Research Paper

\title{
Ferumoxytol- $\beta$-glucan Inhibits Melanoma Growth via Interacting with Dectin-1 to Polarize Macrophages into Ml Phenotype
}

\author{
Xinghan Liu ${ }^{1}$, Yujun $\mathrm{Xu}^{1}$, Yi Li ${ }^{1}$, Yuchen Pan ${ }^{1}$, Shuli Zhao ${ }^{2}$ and Yayi Hou ${ }^{1,3}$ \\ 1. The State Key Laboratory of Pharmaceutical Biotechnology, Division of Immunology, Medical School, Nanjing University, Nanjing 210093, China. \\ 2. General Clinical Research Center, Nanjing First Hospital, Nanjing Medical University, Nanjing 210006, China. \\ 3. Jiangsu Key Laboratory of Molecular Medicine, Nanjing University, Nanjing 210093, China.
}

$\triangle$ Corresponding authors: Yayi Hou, The State Key Laboratory of Pharmaceutical Biotechnology, Division of Immunology, Medical School, Nanjing University, Nanjing 210093, China. Tel.: +86-25-8968-8441; Fax: +86-25-8968-8441. E-mail: yayihou@nju.edu.cn; Shuli Zhao, General Clinical Research Center, Nanjing First Hospital, Nanjing Medical University, Nanjing 210006, China. E-mail: shulizhao79@163.com.

(C) The author(s). This is an open access article distributed under the terms of the Creative Commons Attribution License (https://creativecommons.org/licenses/by/4.0/). See http://ivyspring.com/terms for full terms and conditions.

Received: 2021.04.12; Accepted: 2021.06.14; Published: 2021.06.26

\begin{abstract}
Background: Regulating the polarization of macrophages to antitumor Ml macrophages is a promising strategy for overcoming the immunosuppression of the tumor microenvironment for cancer therapy. Ferumoxytol (FMT) can not only serve as a drug deliver agent but also exerts anti-tumor activity. $\beta$-glucan has immuno-modulating properties to prevent tumor growth. Thus, a nanocomposite of FMT surface-coated with $\beta$-glucan (FMT- $\beta$-glucan) was prepared to explore its effect on tumor suppression.

Methods: Male B16F10 melanoma mouse model was established to explore the antitumor effect of FMT- $\beta$-glucan. The viability and apoptotic rates of B16F10 cells were detected by cell counting kit- 8 and Annexin-V/PI experiments. The levels of MI markers were quantified by quantitative reverse transcription-polymerase chain reaction and enzyme linked immunosorbent assay. Phagocytic activity and intracellular reactive oxygen species (ROS) in macrophages were evaluated by the neutral red uptake assay and flow cytometry, respectively. Small interfering RNA (siRNA) transfection was applied to knock down the Dectin-1 gene in RAW 264.7 cells.

Results: FMT- $\beta$-glucan suppressed tumor growth to a greater extent and induced higher infiltration of $\mathrm{Ml}$ macrophages than the combination of FMT and $\beta$-glucan (FMT+ $\beta$-glucan) in vivo. In vitro, supernatant from FMT- $\beta$-glucan-treated RAW 264.7 cells led to lower cell viability and induced more apoptosis of B16F10 cells than that from the FMT+ $\beta$-glucan group. Moreover, FMT- $\beta$-glucan boosted the expression of MI type markers, and increased phagocytic activity and ROS in RAW 264.7 cells. Further research indicated that FMT- $\beta$-glucan treatment promoted the level of Dectin-1 on the surface of RAW 264.7 cells and that knockdown of Dectin-1 abrogated the phosphorylation levels of several components in MAPK and NF-KB signaling.

Conclusion: The nanocomposite FMT- $\beta$-glucan suppressed melanoma growth by inducing the $\mathrm{Ml}$ macrophage-activated tumor microenvironment.
\end{abstract}

Key words: FMT, $\beta$-glucan, macrophage, Dectin-1, melanoma

\section{Introduction}

The latest estimated data on melanoma showed nearly 100,350 new cases and 6850 deaths occurring in the United States in 2020, as the fifth most common cancer in males and females [1]. The incidence of melanoma has exhibited a continuous increase over the past 40 years, with slight improvement in long-term survival [2]. However, the survival rate of patients with melanoma remains low. After surgical resection, approximately $30 \%$ of the patients develop metastasis in various organs. Patients carrying metastatic melanoma have a bad prognosis, with a five-year survival rate between 5-19\% [3]. Compared with other tumors, melanoma is considered an immunogenic malignancy because of the large number of tumor-infiltrating lymphocytes [4]. Based on these findings, immunotherapies, such as immune 
checkpoint inhibitors and cytokines, have revolutionized the strategies for melanoma treatment [5]. Several drugs, such as ipilimumab, pembrolizumab and bempegaldesleukin have been approved for use in the first-line setting of advanced melanoma [6, 7]. Besides low survival rates for a substantial number of patients, problems such as low clinical response, drug resistance, and high drug prices hinder the implementation of immunotherapies $[8,9]$. Therefore, seeking and developing more effective and practical approaches for melanoma treatment is urgent needed.

Ferumoxytol (FMT), an FDA-approved iron oxide nanoparticle, is widely used to treat iron deficiency anemia [10, 11]. In a previous phase III clinical study, the intravenous iron replacement therapy with FMT was well tolerated in the patients and achieved the expected therapeutic effect without any gastrointestinal symptoms or hypotension [12]. Given its strong safety and unique characterization to label macrophages, FMT has been used to identify inflammation status and monitor therapy-mediated changes in inflammatory diseases in magnetic resonance imaging $[13,14]$. Moreover, the potential value of FMT in tumor therapies has recently gained great interest from researchers. Although the clinical dose of FMT had no direct tumoricidal effect, coinjection of cancer cells and FMT markedly suppressed tumor development and metastasis in the liver and lungs by increasing M1 polarization of macrophages in a murine tumor model [15]. In addition, FMT combined with other immunologic agents (Toll-like receptor (TLR) 3 agonist (poly(I:C)) or TLR 9 agonist $(\mathrm{CpG})$ ) exerted stronger antitumor activity than FMT used alone [16, 17]. Thus, we should explore the synergy of FMT and other immune activators in order to develop more effective treatment strategies for melanoma.

$\beta$-glucan is a type of natural polysaccharide that can be extracted from a variety of plants such as oats, barley, and seaweed and is also the component of the cell walls of some pathogenic bacteria (Pneumocystis carinii, Cryptococcus, Aspergillus fumigatus, and Candia albicans) and fungi (Saccaromyces cerevisiae). Although different kinds of $\beta$-glucan are all glucan polymers connected by $\beta-(1,3)$ and $(1,4)$ or $(1,6)$ glycosidic chains, their diversities of length and branch structures determine the difference of their extraction method and biological activity [18]. Studies have shown that $\beta$-glucan with large, intermediate or low molecular weight, or yeast-derived $\beta$-glucan has biological activity in vivo, however short $\beta$-glucan (molecular weight less than 5000-10000) is normally inactive $[19,20]$. $\beta$-glucan is considered an important anticancer agent because of its regulation of immune cells, anti-angiogenesis, enhanced sensitivity to radiotherapy and chemotherapy, and direct cytotoxicity to tumor cells [21,22]. The in vitro studies found yeast-derived $\beta$-glucan could be conjunct to the lectin sites of complement receptor 3 (CR3) of neutrophils, macrophages, and natural killer (NK) cells and mediate the cytotoxic effects of these cells against tumors opsonized with iC3b [23, 24]. Vaclav Vetvicka et al. find oral administration of Saccharomyces cerevisiae-derived $\beta$-glucan strongly reduced the tumor weight and lung metastasis, and prolonged the overall survival of mice carrying melanoma in vivo via activating NK cells, and enhanced the efficacy of regular chemotherapeutic drugs [25]. In addition, $\beta$-glucan can alter the immune-suppressive tumor microenvironment. On the one hand, $\beta$-glucan can switch monocytic myeloid-derived suppressor cells (M-MDSCs) to antigen presenting cells, resulting in the activation of $\mathrm{CD}^{+}$and $\mathrm{CD}^{+} \mathrm{T}$ cells, and the expression of proinflammatory cytokines (interferon (IFN)- $\gamma$, tumor necrosis factor (TNF)- $\alpha$, and perforins), and further damaging tumor cells [26]. On the other hand, oat- or yeast-derived $\beta$-glucan induces macrophages polarization from the suppressive M2 status to the tumoricidal M1 phenotype by Dectin-1 or TLR2, then activates Th1 type $T$ cells, and finally suppresses the growth of melanoma and lung cancer [27, 28]. Additionally, pretreatment of mice with $\beta$-glucan can establish trained immunity, thereby rewiring the transcriptomics and epigenetics of granulopoiesis and reprogramming neutrophils toward anti-tumor phenotype, and ultimately diminishing tumor growth [29]. In addition to the above functions, $\beta$-glucan can also directly induce apoptosis of some cells and released reactive oxygen species (ROS) will kill tumor cells irrespective of the immune system. Recently, it was reported that water-soluble $\beta$-D-glucan directly reduced hepatocellular carcinoma (HCC) cell growth in vitro and in vivo, and the effect was significantly linked with autophagy blockage by $\beta$-D-glucan and thereby mitochondria dysfunction and excess ROS, which promoted the apoptosis of HCC cells under the lack of nutrition [30].

Consequently, whether the combination of FMT and $\beta$-glucan $(\mathrm{FMT}+\beta$-glucan) or the nanocomposite of FMT surface-coated with $\beta$-glucan (FMT- $\beta$-glucan) has a synergistic effect on the elimination of tumors and the regulation of macrophages prompted us to conduct subsequent research. In this study, we identified that the nanocomposite FMT- $\beta$-glucan effectively inhibited melanoma growth by inducing the polarization of macrophages into proinflammatory M1 macrophages and displayed greater therapeutic benefit than the treatment of FMT 
combined with $\beta$-glucan. Furthermore, by binding to the Dectin-1 receptor on the surface of macrophages, FMT- $\beta$-glucan polarized macrophages into the M1 type partly through activating the mitogen-activated protein kinase (MAPK) and spleen associated tyrosine kinase (Syk)/nuclear factor kappa-B (NF-kB) signaling pathways.

\section{Methods}

\section{Materials}

$\beta$-glucan used in this study was purchased from Sigma (G-5011), which was hereinafter referred to as $\beta$-glucan for short. $\beta$-glucan with a $\beta$ - $(1,3)$-glucan linear structure and a small number of $\beta-(1,6)$-glucan branches was extracted from Saccaromyces cerevisiae and had been used in other researchers' studies [31, 32]. The preparation method and characterization of $\beta$-glucan has been described in the previous studies $[22,33]$, and the purity of $\beta$-glucan is greater than $98 \%$.

\section{Synthesis of FMT surface-coated with $\beta$-glucan (FMT- $\beta$-glucan)}

FMT was prepared by pathways described in previous reports [34]. To prepare FMT- $\beta$-glucan, FMT, $\beta$-glucan (Merck KGaA, Darmstadt, Germany), and 1-ethyl-3-(3-dimethylaminopropyl) carbodiimide hydrochloride (EDC) (J\&K Scientific Ltd, Shanghai, China) were added to appropriate deionized water and stirred overnight at room temperature. Excess $\beta$-glucan was removed by dialyzing with a dialysis bag (300 kDa MWCO) for $48 \mathrm{~h}$. Finally, the mixture was lyophilized into powder and used for subsequent experiments.

\section{Characterization of FMT- $\beta$-glucan}

Infrared absorption spectroscopy was used to confirm the successful binding of $\beta$-glucan to the FMT surface. The content of Fe in FMT was measured by ICP-OES (Optima 8000, PerkinElmer, USA). The particle sizes and zeta potentials of FMT and FMT- $\beta$-glucan were assessed using the dynamic light scattering technique (Malvern, UK). Transmission electron microscopy (TEM; JEM-1200EX, JEOL, Japan) was applied to observe the morphology of FMT and FMT- $\beta$-glucan. To evaluate the stability in diverse $\mathrm{pH}$ conditions and serum, the FMT- $\beta$-glucan was cultured in solutions with several $\mathrm{pH}$ values of 5.5, 6.8 and 7.4 or DMEM medium (Gibco, Carlsbad, CA) containing $10 \%$ fetal bovine serum (FBS; Gibco, Carlsbad, CA) at $37{ }^{\circ} \mathrm{C}$ and the particle sizes were determined by dynamic light scattering technique.

\section{Rhodamine B (RhB)-labeled FMT- $\beta$-glucan}

Briefly, RhB (0.5 mg; Sigma, USA), FMT- $\beta$ - glucan $(20 \mathrm{mg}$ ), and dimethyl sulfoxide (DMSO; J\&K Scientific Ltd) $(5 \mathrm{~mL})$ were mixed and stirred in an Erlenmeyer flask overnight at ambient temperature. Then the mixture was dialyzed with distilled water by a dialysis bag (3.5 kDa MWCO) for $48 \mathrm{~h}$ and lyophilized into powder.

\section{In vitro drug release}

We evaluated the release of $\beta$-glucan from FMT- $\beta$-glucan under various $\mathrm{pH}$ values $(5.5,6.8$ and 7.4) in vitro. First, we prepared the RhB-labeled $\beta$-glucan using the above instruments. Then a dialysis bag (300 kDa MWCO) with $5 \mathrm{~mL}$ of FMT- $\beta$-glucan solution was placed in a PBS solution and shaken in the dark using a $37^{\circ} \mathrm{C}$ shaker. Finally, the dialysate was removed at selected time points, and the release profile was assessed by fluorescence.

\section{Mouse tumor model}

All of the animal experiment protocols had reached the approval of the Institutional Animal Care and Use Committee of Nanjing First Hospital, Nanjing Medical University (DW20200204) and were conformed to the guides issued by the National Institutes of Health. Male C57BL/6 mice (5-6 weeks old) were bought from the Nanjing Model Animal Institute (Nanjing, China). A subcutaneous tumor mouse model was constructed by subcutaneously injected with $100 \mu \mathrm{L}$ sterile PBS containing $5 \times 10^{5}$ B16F10 cells in the right flank of mice. Mice were randomly distributed into five groups when tumors reaching a volume of about $100 \mathrm{~mm}^{3}$, and intraperitoneally administrated with PBS, FMT (400 $\mu \mathrm{g}), \beta$-glucan $(80 \mu \mathrm{g}), \mathrm{FMT}+\beta$-glucan $(400 \mu \mathrm{g}$ FMT + $80 \mu \mathrm{g} \beta$-glucan) and FMT- $\beta$-glucan, containing FMT $400 \mu \mathrm{g}$ and $\beta$-glucan $80 \mu \mathrm{g}$, every day. Tumor growth was observed via magnetic resonance imaging (MRI; Biospec 7T/20USR GmbH Bruker, Germany). The parameters were set as follows: $\mathrm{TR}=2500 \mathrm{~ms}, \mathrm{TE}=33$ $\mathrm{ms}, \mathrm{FA}=180 \mathrm{deg}$, and field of view $(\mathrm{FOV})=3.5 \mathrm{~cm}$. Body weight and tumor size were measured and recorded every other day by scales and Vernier calipers. The following formula was applied to calculate the tumor volume: volume $(\mathrm{V})=$ length $(\mathrm{L}) \times$ width $(W)^{2} \times 1 / 2$.

\section{Analysis of tumor-infiltrating immunocytes}

Scissors and tweezers were used to carefully peel off the tumor tissues. After weighing, tumor tissues were harvested and digested with RPMI 1640 medium containing 5\% FBS, $2 \mathrm{mg} / \mathrm{mL}$ collagenase IV (Sigma, USA) and $5 \mathrm{U} / \mathrm{mL}$ DNase I (Sigma, USA) for 20 minutes by a $37^{\circ} \mathrm{C}$ shaker. Then the cell suspension was neutralized with RPMI 1640 medium containing $5 \%$ FBS and filtered through 70- $\mu \mathrm{m}$ nylon mesh into a centrifuge tube to obtain single cell suspension. Bone 
marrow cells were obtained as described above. Spleens were milled and filtered with PBS into centrifuge tubes through a 200-mesh sieve. Then, the cells were lysed with red blood cell lysate, filtered through gauze, and resuspended in PBS. For immunocyte detection, the following anti-mouse antibodies were used: CD45-PE (103106, BioLegend), CD45-APC (17-0451-83, eBioscience), CD11b-APC (101212, BioLegend), CD11b-PerCP (45-0112-82, eBioscience), Gr-1-FITC (108406, BioLegend), F4/80-FITC (11-4801-82, eBioscience), NOS2-PE (12-5920-82, eBioscience), CD206-PE (12-2061-82, eBioscience), and CD206-APC (17-2061-82, eBioscience). A FACSCalibur flow cytometer (Becton Dickinson, Franklin Lakes, NJ) was applied to detect the stained cells and the FlowJo software (TreeStar, Ashland, OR) was utilized to analyze the data.

\section{Immunohistochemistry (IHC)}

Harvested tumor tissues were kept in the 4\% paraformaldehyde solution (Servicebio, Wuhan, China) and then embedded in paraffin. Antibodies against Ki67, F4/80, CD86 and CD206 antibody were used as primary antibodies. All sections were imaged via a light microscope (ECLIPSE Ti-U, Nikon, Japan), and the ImageJ software (National Institutes of Health, USA) was used for the analysis of images.

\section{Enzyme-linked immunosorbent assay (ELISA)}

The supernatants of RAW 264.7 cells or blood from mice were collected to determine TNF-a or interleukin (IL)- 6 expression by a commercial mouse TNF-a ELISA kit (4A BiotechCo. Ltd, Beijing, China) and a mouse IL-6 ELISA kit (Neobioscience, Shanghai, China), following the instructions in the manual.

\section{Cell culture and chemicals}

RAW 264.7 cells, B16F10 melanoma cells and ID8 mouse ovarian cancer cells were obtained from Fudan Cell Bank and kept in Dulbecco's modified Eagle's medium (DMEM) or RPMI 1640 medium containing $10 \% \mathrm{FBS}$ and $1 \% \mathrm{v} / \mathrm{v}$ penicillin and streptomycin (Gibco, Carlsbad, CA) at $37{ }^{\circ} \mathrm{C}$ in an incubator with $5 \% \mathrm{CO}_{2}$.

\section{Cell viability}

The cell viability was detected by a Cell Counting Kit-8 (CCK-8) following the instructions in the manual [35]. RAW 264.7 cells, B16F10 and ID8 cells were cultured in 96-well plates at appropriate densities (for the detection of cell viability in a coculture system, B16F10/ID8 and RAW 264.7 cells were mixed in a 1:2 ratio) and treated with FMT, $\beta$-glucan, or a combination of both for $48 \mathrm{~h}$.

\section{Cell proliferation analysis}

First, B16F10 and ID8 cells, and RAW 264.7 cells were prelabeled with the dye CFSE (DOJINDO, Shanghai, China) and eFluor 670 (eBioscience) following the manufacturer's instructions, respectively. Then tumor cells and RAW 264.7 cells were directly or indirectly cocultured at the ratio of $1: 2$ and treated with $400 \mu \mathrm{g} / \mathrm{mL}$ FMT, $80 \mu \mathrm{g} / \mathrm{mL}$ $\beta$-glucan and $400 \mu \mathrm{g} / \mathrm{mL} \mathrm{FMT}+80 \mu \mathrm{g} / \mathrm{mL} \beta$-glucan for $48 \mathrm{~h}$. For cell proliferation analysis, the cells were analyzed by FCM. Mod Fit LT 3.0 software was applied to calculate the proliferation index (PI) of tumor cells. The growth inhibition rate of tumor cells was measured according to the following equation: cell growth inhibition rate $=(1-(\mathrm{PI}$ (treatment group $) /$ PI (control group) ) $\times 100 \%$.

\section{Cell apoptosis analysis}

B16F10 cells were treated with $\mathrm{FMT}+\beta$-glucan and FMT- $\beta$-glucan for $48 \mathrm{~h}$. Then the cells were harvested and incubated with staining solution that comprised of $195 \mu \mathrm{L}$ binding buffer, $5 \mu \mathrm{L}$ Annexin $\mathrm{V}$, and $2.5 \mu \mathrm{L}$ PI for 15 minutes at ambient temperature without light. Finally, the cells were resuspended in binding buffer solution and subjected to flow cytometry (FCM) analysis.

\section{Quantitative reverse transcription-polymerase chain reaction ( $q R T-P C R$ )}

TRIzol reagent (Invitrogen, Grand Island, NY, USA) was employed to obtain total RNA from treated cells. Then, $1 \mu \mathrm{g}$ of the collected RNA was reversetranscribed to cDNA following the protocol of a HiScript II Q RT SynthesisMix Kit (Vazyme, Nanjing, China), and cDNA was subjected to PCR quantification by SYBR Green SuperMix reagent (Bio-Rad, California, USA). Finally, the levels of genes of interest were quantified by the $2-\Delta \Delta \mathrm{Ct}$ method based on its relative expression to GAPDH. Detail sequences of the primers are available in Table 1.

\section{Neutral red uptake assay}

Treated cells cultured in a 96-well plate were stimulated for $1 \mathrm{~h}$ with $200 \mu \mathrm{L} /$ well physiological saline solution containing $0.1 \%$ neutral red and washed with mild phosphate buffered solution (PBS) for 3 times. Then $200 \mu \mathrm{L}$ cell lysate prepared in an equal volume of absolute ethanol and acetic acid was added into each well, and the cells were set aside for at least $3 \mathrm{~h}$ at room temperature. Finally, the absorbance values of each well were examined at 540 nm. 
Table 1. Primer sequences used in qRT-PCR

\begin{tabular}{|c|c|}
\hline Gene name & Primer sequence $\left(5^{\prime}\right.$ to $\left.3^{\prime}\right)$ \\
\hline \multicolumn{2}{|l|}{ GAPDH } \\
\hline forward & AGGTCGGTGTGAACGGATTTG \\
\hline reverse & TGTAGACCATGTAGTTGAGGTCA \\
\hline \multicolumn{2}{|l|}{ TNF- $\alpha$} \\
\hline forward & CAGGCGGTGCCTATGTCTC \\
\hline reverse & CGATCACCCCGAAGTTCAGTAG \\
\hline \multicolumn{2}{|l|}{ iNOS } \\
\hline forward & GGAGTGACGGCAAACATGACT \\
\hline reverse & TCGATGCACAACTGGGTGAAC \\
\hline \multicolumn{2}{|l|}{ IL-1 $\beta$} \\
\hline forward & GAAATGCCACCTTTTGACAGTG \\
\hline reverse & TGGATGCTCTCATCAGGACAG \\
\hline \multicolumn{2}{|r|}{ 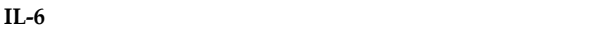 } \\
\hline forward & AAGAAATGATGGATGCTACC \\
\hline reverse & AGTTTCTGTATCTCTCTGAAG \\
\hline \multicolumn{2}{|l|}{ Arg-1 } \\
\hline forward & CTCCAAGCCAAAGTCCTTAGAG \\
\hline reverse & GGAGCTGTCATTAGGGACATCA \\
\hline \multicolumn{2}{|l|}{ CD206 } \\
\hline forward & СTCTGTTCAGCTATTGGACGC \\
\hline reverse & TGGCACTCCCAAACATAATTTGA \\
\hline \multicolumn{2}{|l|}{ p22phox } \\
\hline forward & CTACTGCTGGACGTTTCACAC \\
\hline reverse & GGTGGACCССTTTTTCСТCTT \\
\hline \multicolumn{2}{|l|}{ p40phox } \\
\hline forward & GGGCCATCATGGATCGCAT \\
\hline reverse & CCAGCCAGTCTTTGTTGATCTT \\
\hline \multicolumn{2}{|l|}{ p47phox } \\
\hline forward & ACACCTTCATTCGCCATATTGC \\
\hline reverse & CCTGCCACTTAACCAGGAACA \\
\hline \multicolumn{2}{|l|}{ p67phox } \\
\hline forward & GGAGAAGTACGACCTTGCTATCA \\
\hline reverse & ACAGGCAAACAGCTTGAACTG \\
\hline \multicolumn{2}{|l|}{ NOX2 } \\
\hline forward & AGTGCGTGTTGCTCGACAA \\
\hline reverse & GCGGTGTGCAGTGCTATCAT \\
\hline \multicolumn{2}{|r|}{ ( } \\
\hline forward & ACAGTAAGCCGGTGAACCTG \\
\hline reverse & CTGACTAGCGAGAAGCAGATG \\
\hline Bcl-2 & \\
\hline forward & GCTACCGTCGTGACTTCGC \\
\hline reverse & CCCCACCGAACTCAAAGAAGG \\
\hline Bax & \\
\hline forward & AGACAGGGGCCTTTTTGCTAC \\
\hline reverse & AATTCGCCGGAGACACTCG \\
\hline CDK2 & \\
\hline forward & ATGGAGAACTTCCAAAAGGTGG \\
\hline reverse & CAGTCTCAGTGTCGAGCCG \\
\hline CDK4 & \\
\hline forward & ATGGCTGCCACTCGATATGAA \\
\hline reverse & TGCTCCTCCATTAGGAACTCTC \\
\hline cyclin A2 & \\
\hline forward & GCCTTCACCATTCATGTGGAT \\
\hline reverse & TTGCTCCGGGTAAAGAGACAG \\
\hline cyclin B1 & \\
\hline forward & CTTGCAGTGAGTGACGTAGAC \\
\hline reverse & CCAGTTGTCGGAGATAAGCATAG \\
\hline Dectin-1 & \\
\hline forward & AAAGCCAAACATCGTCTCACC \\
\hline reverse & CGAGTTGGGGAAGAATGCTGAT \\
\hline Dectin-2 & \\
\hline forward & TCCACAAGGTAATGGCAAATGG \\
\hline reverse & CTATTGAAACACACCGCTCTTCT \\
\hline Dectin-3 & \\
\hline forward & ACCCGACATCCCCAACTGAT \\
\hline reverse & CTCTCGTCCAGCGTAAAAAGT \\
\hline mincle & \\
\hline forward & TGTCGTAACATATCGCAGCTC \\
\hline reverse & GGACAGCAATTCTTGACTGAACC \\
\hline
\end{tabular}

Detection of reactive oxygen species (ROS)

After stimulation for $12 \mathrm{~h}$, cell culture supernatants were discarded and an appropriate volume of serum-free medium with a final concentration of $10 \mu \mathrm{M}$ of 2,7-dichlorodihydrofluorescein diacetate (DCFH-DA) (Beyotime, Shanghai, China) was added. Then the cells were incubated for 20 minutes at $37^{\circ} \mathrm{C}$ without light and washed in serum-free medium for 3 times to fully remove the left DCFH-DA. Finally, the cells were subjected to FCM analysis.

\section{Western blotting (WB) analysis}

RIPA lysis buffer (Beyotime, Shanghai, China) was utilized to obtain the total proteins. After being quantified by a BCA protein assay kit (Thermo Fisher Scientific, Waltham, MA), equal proteins were loaded and electrophoresed on a polyacrylamide gel, and wet-transferred to PVDF membranes (Millipore, Bedford, MA). Then the membranes were kept in 5\% bovine serum albumin (BSA) in Tris-buffered saline with $0.1 \%$ Tween 20 (TBST) at indoor temperature for $1.5 \mathrm{~h}$ and kept in primary antibody solution with a 1:1000 dilution ratio at $4{ }^{\circ} \mathrm{C}$ for $12 \mathrm{~h}$. After being treated with secondary antibody solution with a 1:5000 dilution ratio for $1 \mathrm{~h}$ at ambient temperature, the membranes were observed with ECL Plus WB detection reagents (Millipore).

The primary antibodies including mouse anti-phosphorylated (p)-p38, mouse anti-p38, rabbit anti-p-extracellular regulated MAP kinase (p-ERK), rabbit anti-ERK, rabbit anti-p-c-Jun NH2-terminal (p-JNK), rabbit anti-JNK, rabbit anti- $\beta$-actin, rabbit anti-p-Syk, rabbit anti-Syk, rabbit anti-p- protein kinase $\mathrm{C}$ delta (PKC $\delta$ ), rabbit anti-PKC $\delta$, rabbit anti-p-IKK $\alpha / \beta$, rabbit anti-IKK $\alpha$, rabbit anti-p-P65, and rabbit anti-P65 were bought from Cell Signaling Technology (Danvers, Massachusetts, USA). The rabbit anti-Histone 3 antibody was bought from Abcam (Cambridge, Massachusetts, USA).

\section{Immunofluorescence staining}

The transportation of P65 to the nucleus was detected by immunofluorescence staining to confirm whether NF-kB pathway was activated, according to the protocol of a nuclear translocation assay kit (Beyotime, Shanghai, China), as described previously [36].

\section{Uptake of FMT- $\beta$-glucan by macrophages in vitro}

RAW 264.7 cells were treated with RhB-labeled FMT- $\beta$-glucan for $6 \mathrm{~h}$ or $24 \mathrm{~h}$ and the uptake of FMT- $\beta$-glucan by RAW 264.7 cells was observed by FCM or FV3000 laser scanning confocal microscopy 
(LSCM; lympus, Japan) after staining the nuclei with 4,6-diamino-2-phenyl-indole (DAPI) for 5 minutes.

\section{Small interfering RNA (siRNA) transfection}

siRNA oligonucleotides for Dectin-1 and a noncoding (NC) siRNA with no biological effects were obtained from GenePharma (Shanghai, China). siRNA transfection was performed according to the product instructions at a concentration of $50 \mathrm{nM}$. After transfection for $24 \mathrm{~h}$ and $48 \mathrm{~h}, \mathrm{qRT}-\mathrm{PCR}$ and WB assays were used to detect the knockdown efficiency of Dectin-1.

\section{Statistical analysis}

Prism 6 (GraphPad Software, Inc., San Diego, CA) was applied to perform the statistical analysis by Student's t-test or one-way ANOVA. Significant differences were marked as ${ }^{*} p<0.05$ and $^{* *} p<0.01$.

\section{Results}

\section{$F M T+\beta$-glucan has an inhibitory effect on tumor growth by regulating macrophages in vivo}

First, we assessed the effect of FMT, $\beta$-glucan and their combination (FMT $+\beta$-glucan) on tumor growth in vivo of male melanoma bearing C57BL/ 6 mice. As depicted in Figure $1 \mathrm{~A}-\mathrm{C}$, intraperitoneal injection of FMT or $\beta$-glucan remarkably delayed tumor progression via reduced tumor weight and tumor volume, and coadministration of FMT plus $\beta$-glucan significantly improved the therapeutic effect. Immunohistochemical analysis of Ki67 in tumor tissues also found that FMT or $\beta$-glucan treatment reduced tumor proliferation, and co-administration notably amplified the effect (Figure $1 \mathrm{D}$ and $1 \mathrm{E})$. Furthermore, the Prussian blue staining showed that FMT $+\beta$-glucan could accumulate in the tumor tissues (Figure 1G).

Previous studies reported that FMT or $\beta$-glucan might induce macrophage polarization in vitro and in vitro, thus the distribution and subgroups of macrophages in tumor tissues, bone marrow cells and splenocytes were examined. FCM results indicated that treatment with FMT $+\beta$-glucan did not alter the infiltration of MDSCs $\left(\mathrm{CD} 45^{+} \mathrm{CD} 11 \mathrm{~b}^{+} \mathrm{Gr}-1^{+}\right)$compared to that in the control group and solo treatment, but increased the ratio of total macrophages $\left(\mathrm{CD} 11 \mathrm{~b}^{+} \mathrm{F} 4 / 80^{+}\right.$in $\mathrm{CD}^{+} 5^{+}$cells) and M1-type macrophages in tumor tissues (Figure 1H-L). M2-like macrophages were significantly decreased in any treatment groups (Figure 1M). Meanwhile, a significant difference was also found in bone marrow cells and splenocytes by administration of FMT $+\beta$-glucan. The percentage of total macrophages in bone marrow cells and the ratio of total and M1 phenotype macrophages in splenocytes increased markedly, while the proportion of M2-type macrophages sharply decreased in both (Figure S1). In addition, systemic administration of $\mathrm{FMT}+\beta$-glucan increased the expression of TNF- $\alpha$ in the blood serum of mice (Figure $1 \mathrm{~F}$ ). Therefore, these results together demonstrated that $\mathrm{FMT}+\beta$-glucan resulted in a macrophage-activated tumor microenvironment, which possibly contributed to durable melanoma regression.

\section{Preparation and characteristics of FMT- $\beta$-glucan}

For simultaneous delivery of FMT and $\beta$-glucan, an engineered nanocomposite (FMT- $\beta$-glucan) was prepared. As observed in Figure 2A, the surface of FMT was successfully coated with $\beta$-glucan. The particle size and zeta potential of FMT- $\beta$-glucan, detected by dynamic light scattering, can be found in Figure 2B and 2C. FMT and FMT- $\beta$-glucan nanoparticles were homogeneously dispersed as revealed by TEM (Figure 2D). We then assessed the stability of FMT- $\beta$-glucan at various $\mathrm{pH}$ conditions by measuring particle sizes. The particle size of FMT- $\beta$-glucan increased slightly when the $\mathrm{pH}$ value of the solution decreased from $\mathrm{pH} 7.4$ to 5.5, suggesting that the nanocomposite might release $\beta$-glucan and FMT under the acidic conditions (Figure $2 \mathrm{E})$. We further assessed the release profile of $\beta$-glucan from FMT- $\beta$-glucan in vitro and found that $\beta$-glucan was released faster in the $\mathrm{pH} 6.8$ medium than in $\mathrm{pH} 7.4$ medium (Figure 2F). Under the $\mathrm{pH} 5.5$ conditions, approximately $50 \%$ of $\beta$-glucan was released from the nanocomposite after $24 \mathrm{~h}$ and $90 \%$ was released after $72 \mathrm{~h}$ (Figure $2 \mathrm{~F}$ ). In addition, the particle sizes of FMT- $\beta$-glucan had no obvious change during $24 \mathrm{~h}$ incubation with 10\% FBS solution, which indicated that MT- $\beta$-glucan was suitable for systemic injection (Figure $2 \mathrm{G}$ ).

\section{FMT- $\beta$-glucan displays a more notable inhibitory effect on tumor growth than by $F M T+\beta$-glucan by regulating macrophages in vivo}

Encouraged by the above results, whether the synthesized nanocomposite (FMT- $\beta$-glucan) had the same efficacy of tumor elimination as the combination treatment of FMT $+\beta$-glucan was then explored in vivo. We surprisingly found that FMT- $\beta$-glucan displayed a stronger tumor growth inhibition effect than FMT $+\beta$-glucan with reduced tumor weight and tumor volume, as well as decreased Ki67 expression (Figure $3 \mathrm{~A}-3 \mathrm{E})$. In addition, the Prussian blue staining showed that FMT- $\beta$-glucan had significantly higher FMT accumulation in tumor tissues than 
FMT $+\beta$-glucan (Figure 3G). Given the polarization of macrophages induced by FMT $+\beta$-glucan, the efficacy of FMT- $\beta$-glucan was also detected. Consistent with the previous results, FMT- $\beta$-glucan treatment also increased the infiltration of macrophages especially M1-type macrophages and decreased M2 counterparts to a greater extent than $\mathrm{FMT}+\beta$-glucan by IHC experiment (Figure $3 \mathrm{~F}$ and $3 \mathrm{H}$ ). ELISA experiment revealed the elevated TNF- $\alpha$ expression in the blood serum of mice administrated with FMT- $\beta$-glucan (Figure 3I). Therefore, these results together proved that FMT- $\beta$-glucan might have a better antimelanoma effect than the combination of FMT plus $\beta$-glucan.
A

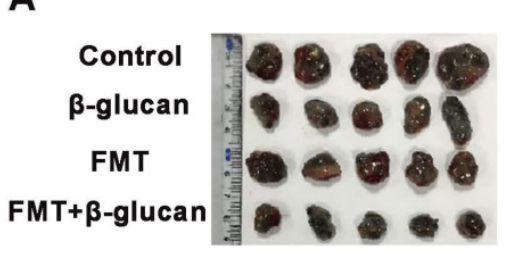

D

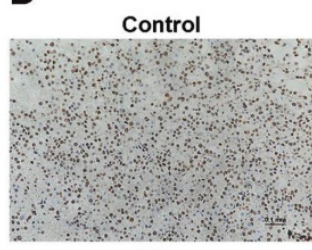

FMT

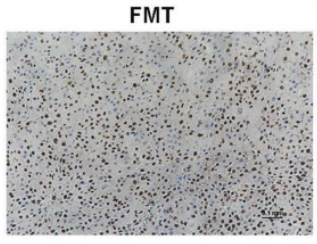

G

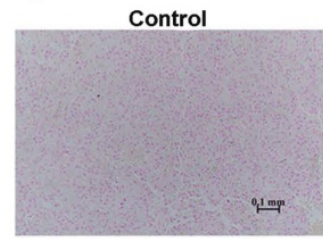

FMT

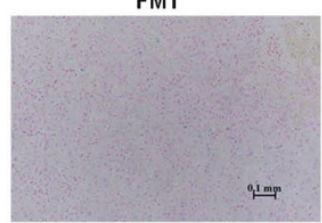

J

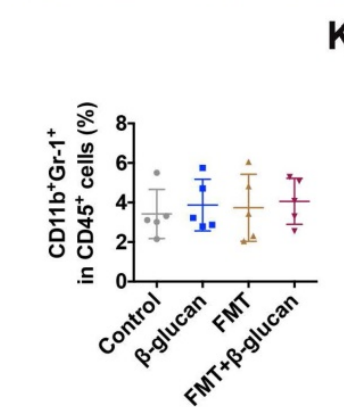

K
B
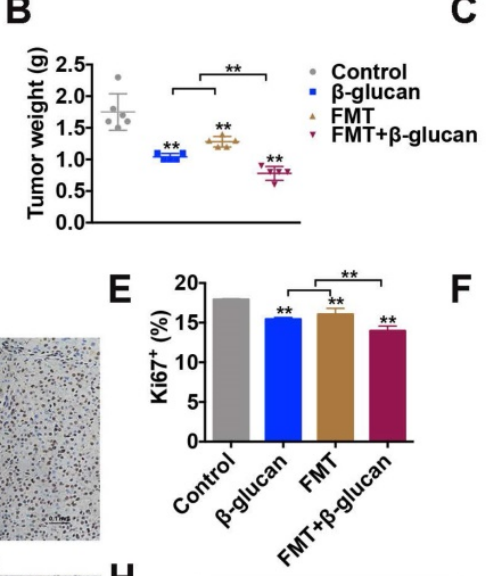

H

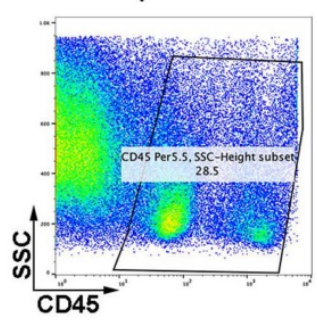

F
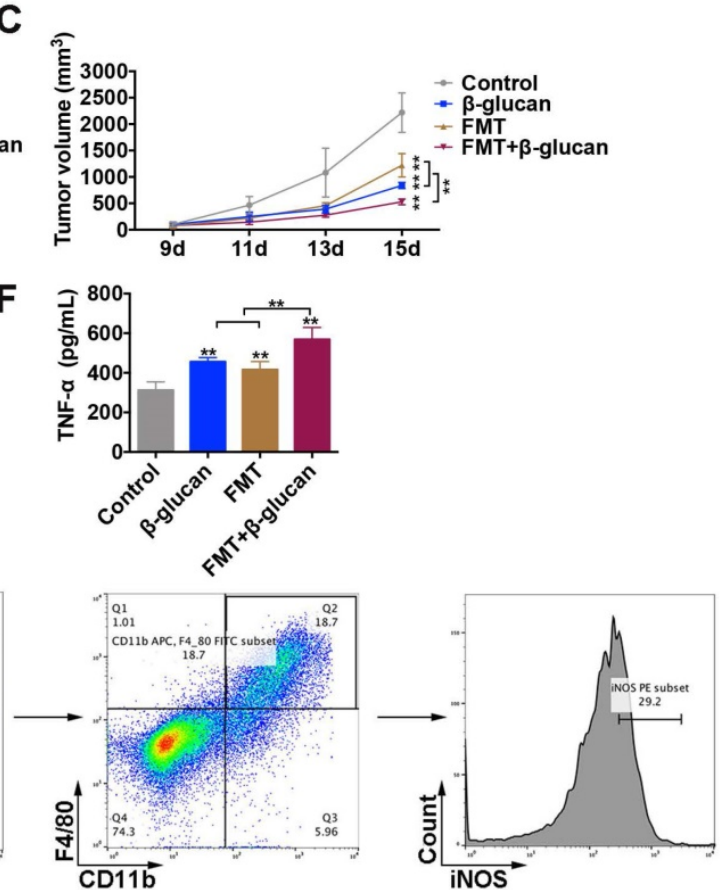$$
\text { . }
$$
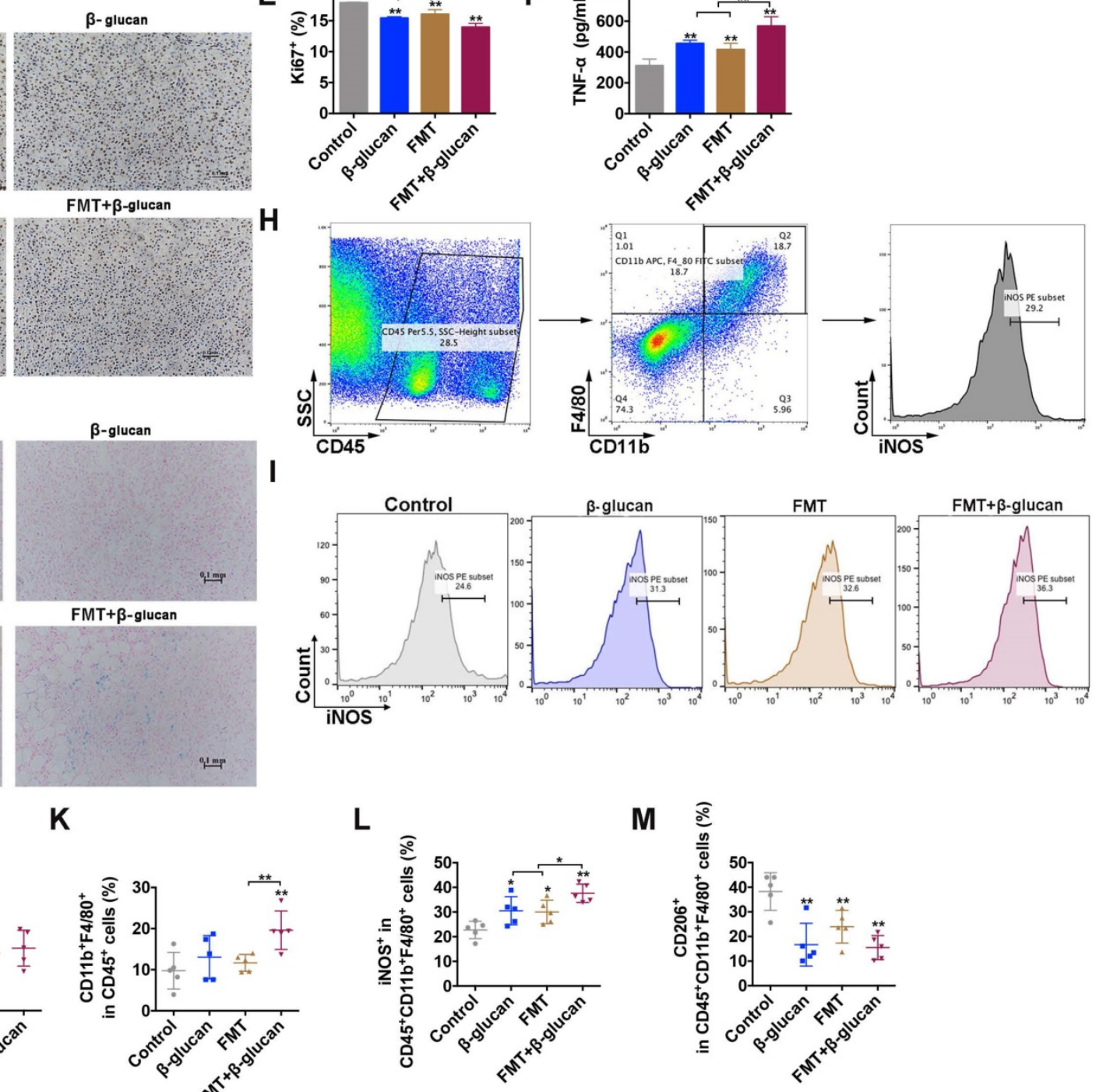

Figure 1. FMT+ $\beta$-glucan suppressed tumor growth and regulated macrophages in vivo. (A) Photograph of dissected tumor tissues from mice with the indicated treatments. (B) Tumor weight of dissected tumor tissues from mice with the indicated treatments. (C) Tumor volume of mice during the indicated treatment. (D) Histopathologic photograph of Ki67 staining of tumor tissues from mice with the indicated treatments. (E) Quantitative data of D. (F) The expression of TNF- $\alpha$ in the blood serum of mice with the indicated treatments. $(G)$ Images of Prussian blue staining of dissected tumor tissues from mice with the indicated treatments. (H) Schematic diagram of the gating principle in the FCM. (I) FCM photographs of iNOS expression in the tumor tissues with the indicated treatment. (J-M) Quantitative data of FCM analysis of MDSCs (CD45+CD11 b+Gr-1+;

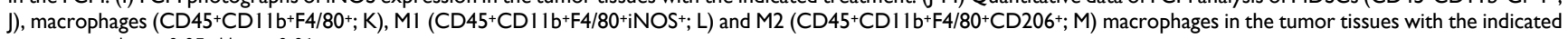
treatments. ${ }^{*} \mathrm{p}<0.05, * * \mathrm{p}<0.01$. 
A

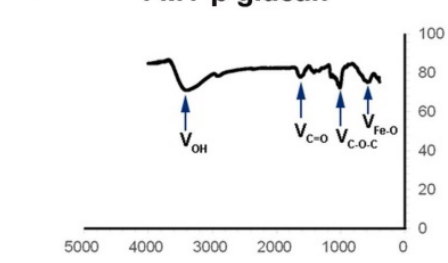

D

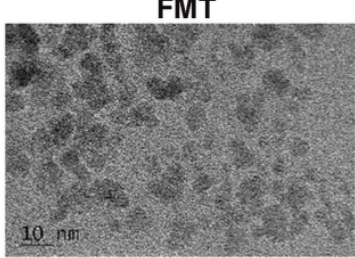

FMT- $\beta$-glucan
B

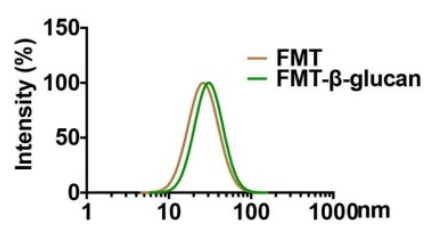

E

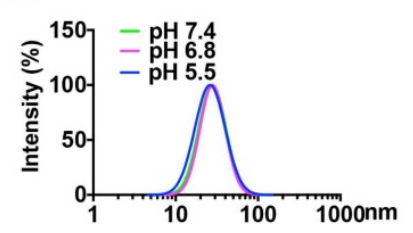

G

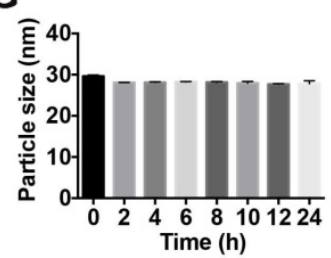

C
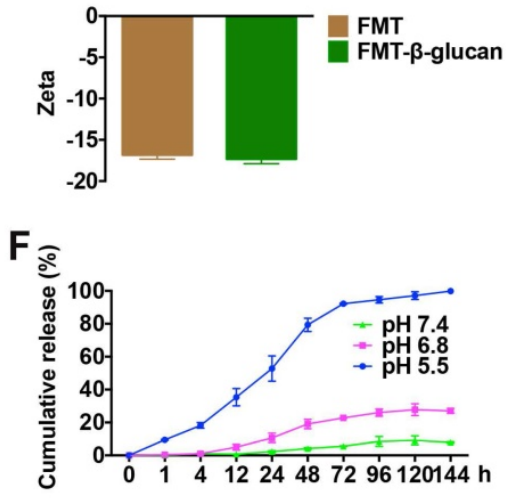

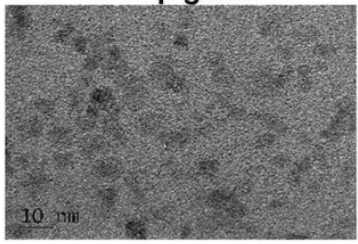

Figure 2. Characteristics of FMT- $\beta$-glucan. (A) The successful binding of $\beta$-glucan to the FMT surface was confirmed by infrared absorption spectroscopy. (B) Particle size of FMT and FMT- $\beta$-glucan. (C) Zeta potential of FMT and FMT- $\beta$-glucan. (D) Images of FMT and FMT- $\beta$-glucan were obtained by TEM. (E) Changes in the particle size of FMT- $\beta$-glucan at different $\mathrm{pH}$ conditions. (F) Release profiles of $\beta$-glucan from FMT- $\beta$-glucan under different $\mathrm{pH}$ conditions. (G) Particle sizes of FMT- $\beta$-glucan after incubation for different durations in DMEM supplemented with $10 \%$ FBS at $37^{\circ} \mathrm{C}$.

\section{Effect of FMT- $\beta$-glucan on tumor growth inhibition in vitro}

Considering the function of FMT- $\beta$-glucan in tumor suppression and macrophage regulation in vivo, the effect of FMT- $\beta$-glucan in vitro was determined. First, the synergistic therapeutic role of the combination treatment of FMT and $\beta$-glucan was studied. The effects of FMT, $\beta$-glucan, and FMT $+\beta$ glucan on B16F10 cell viability were determined by incubation for $48 \mathrm{~h}$. No obvious toxicity in any treatment was observed (Figure 4A). The CCK-8 results showed that when B16F10 and RAW 264.7 cells were cocultured, the addition of $\beta$-glucan and FMT $+\beta$-glucan would reduce cell viability by $6.12 \%$ and $12.2 \%$, respectively (Figure 4B). To accurately verify the direct and indirect inhibition rates on B16F10 cell proliferation, B16F10 cells and RAW 264.7 cells were prestained with dyes CFSE and eFluor 670, respectively. As shown in Figure 4C and Figure 4D, in the direct coculture system of B16F10 and RAW 264.7 cells, treatment with FMT or $\beta$-glucan only slightly suppressed B16F10 cell growth (19.37\% and 10.32\%), whereas the additional FMT in the $\beta$-glucan-treated system contributed to a more notable growth inhibition of B16F10 cells (37.29\%). In the indirect coculture system (Figure 4E), stimulation with FMT or $\beta$-glucan or $\mathrm{FMT}+\beta$-glucan resulted in cell growth inhibition rates of $13.04 \%, 8.33 \%$, and $27.36 \%$, which were lower than the effect of direct coculture (Figure $4 \mathrm{~F}$ and $4 \mathrm{G})$. In addition, the supernatant collected from RAW 264.7 cells (MФs) without or with any stimulation was used to treat B16F10 cells for $48 \mathrm{~h}$. As seen in the Figure $4 \mathrm{H}$, in the presence of FMT $+\beta$-glucan MФs, B16F10 only obtained a cell viability of $54.91 \%$, which was lower than that in FMT

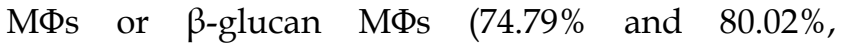
respectively). Next, the ID8 cells were cocultured with RAW 264.7 cells under conditions similar to those described above, and similar results were found (Figure S2).

Then, the therapeutic potential of FMT- $\beta$-glucan on B16F10 cells was examined. FMT $+\beta$-glucan was used for comparison. Compared with the FMT $+\beta$-glucan group, FMT- $\beta$-glucan treatment revealed a more significant reduction in cell viability in the coculture system of B16F10 and RAW 264.7 cells by CCK-8 assay (Figure 4I). The decrease in cell viability could be caused by multiple factors, such as cell cycle arrest and apoptosis. The cell cycle is often dysregulated in tumors and therapies targeting the cell cycle have become an intense subject of research in recent years [37]. Apoptosis is a type of programmed cell death, which can be mediated by the caspase-related pathways [38]. Thus, FCM, qRT-PCR and WB assays were used to detect changes in the cell cycle and apoptosis. The supernatants of RAW 264.7 cells treated with FMT $+\beta$-glucan and FMT- $\beta$-glucan were collected and used to incubate B16F10 cells. Supernatants from the FMT- $\beta$-glucan group (FMT- $\beta$-glucan MФs) reduced B16 F10 cell viability to a greater extent $(31.95 \%)$ and induced more apoptosis 
$(51.23 \%)$ than those in the $\mathrm{FMT}+\beta$-glucan group (51.18\% and $23.05 \%$, respectively) (Figure $4 \mathrm{~J}-\mathrm{L}$ ). As displayed in Figure 4M, decreased cell viability was tightly correlated with the reduction of the ratio of Bcl-2 to Bax and cyclin-dependent kinases (CDK)2, CDK4, cyclin A2, and cyclin B1. In conclusion, although FMT- $\beta$-glucan had no direct tumoricidal effect on tumor cells, FMT- $\beta$-glucan might suppress tumor growth by activating macrophages.

\section{FMT- $\beta$-glucan promoted M1 polarization, and increased phagocytosis and ROS production in macrophages}

To verify the internalization of FMT- $\beta$-glucan by macrophages, FMT- $\beta$-glucan was fluorescently labeled with $\mathrm{RhB}$, and the cellular uptake of
FMT- $\beta$-glucan was quantified by confocal microscopy and FCM (Figure 5A-5B). Classic M1 type macrophages exert tumoricidal activities partly by secreting cytokines (e.g., tumor necrosis factor (TNF)- $\alpha$, IL-1 $\beta$ and IL-6), and reactive species of oxygen and nitrogen (e.g., nitric oxide and superoxide) [39]. Thus, to investigate the potential role of FMT- $\beta$-glucan in polarizing RAW 264.7 cells, the expression of M1-like genes was detected by qRT-PCR after cells were stimulated for $6 \mathrm{~h}$. Compared with controls and FMT $+\beta$-glucan, FMT- $\beta$-glucan significantly promoted the expression of M1-like genes (TNF- $\alpha$, iNOS, IL-1 $\beta$, and IL-6) (Figure 5C) and secretion of TNF- $\alpha$ and IL-6 in the supernatants by ELISA assay (Figure 5D).
A

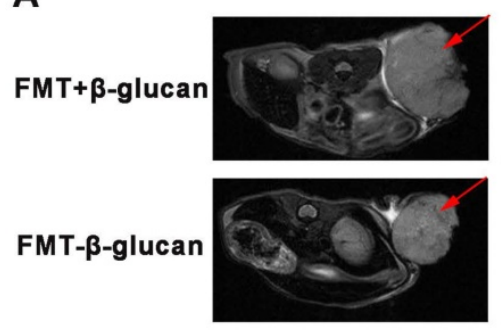

D

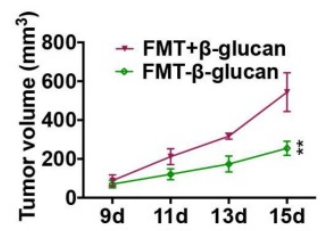

G

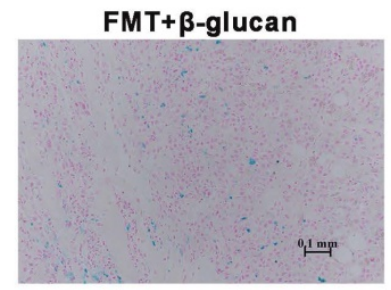

H

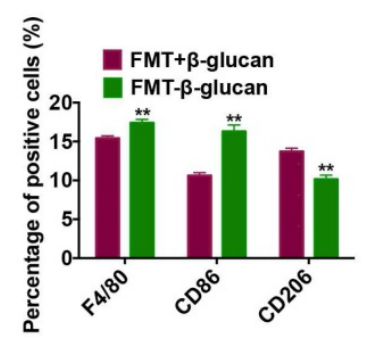

B

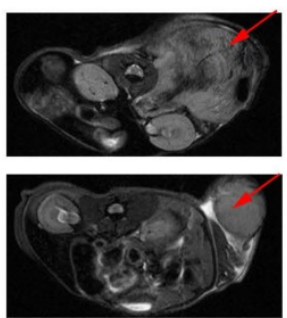

E

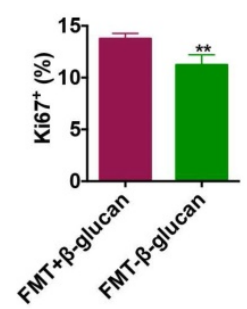

FMT- $\beta$-glucan

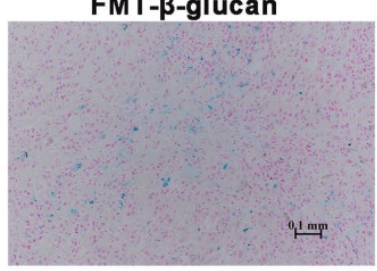

I

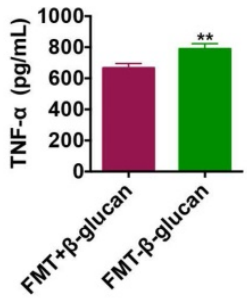

C

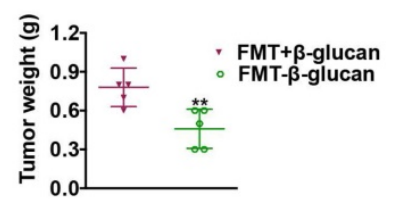

F

Ki67

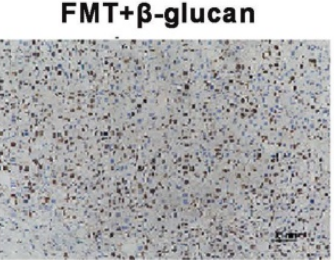

FMT- $\beta$-glucan

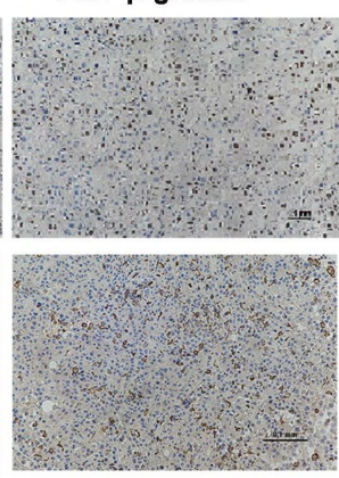

CD86
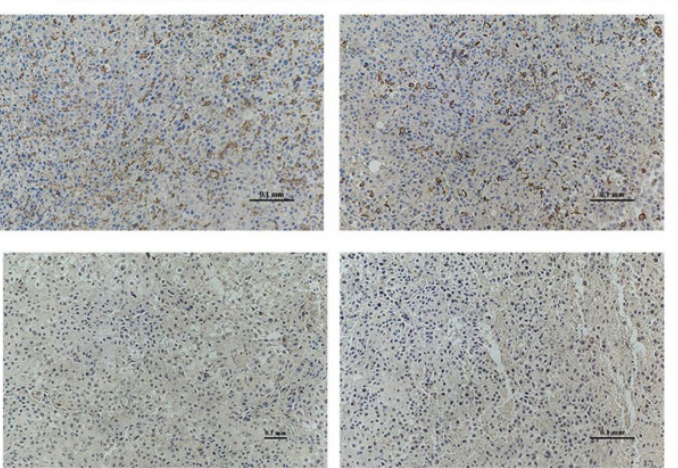

CD206
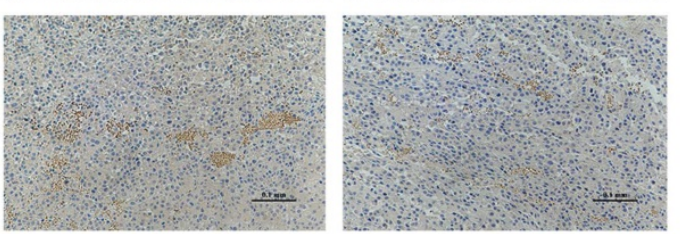

Figure 3. FMT- $\beta$-glucan suppressed tumor growth to a greater extent than $F M T+\beta$-glucan by polarizing macrophages in vivo. (A) MRI images of mice with FMT+ $\beta$-glucan or FMT- $\beta$-glucan treatment. (B) Photograph of dissected tumor tissues from mice with the indicated treatments. (C) Tumor weight of dissected tumor tissues from mice with the indicated treatments. (D) Tumor volume of mice during the indicated treatments. (E) Quantitative data of Ki67 staining of tumor tissues from mice with the indicated treatments. (F) Histopathologic photographs of Ki67, F4/80, CD86 and CD206 staining of tumor tissues from mice with the indicated treatments. (G) Images of Prussian blue staining of dissected tumor tissues from mice with the indicated treatments. (H) Quantitative data of F4/80, CD86 and CD206 staining of tumor tissues from mice with the indicated treatments. (I) The expression of TNF- $\alpha$ in the blood serum of mice with the indicated treatments. ${ }^{*} \mathrm{p}<0.05, * * \mathrm{p}<0.01$. 


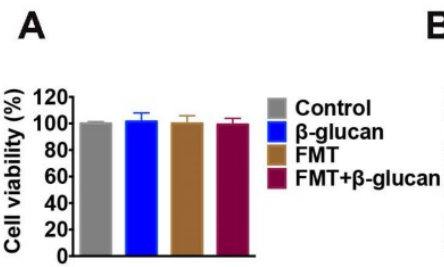

B

E

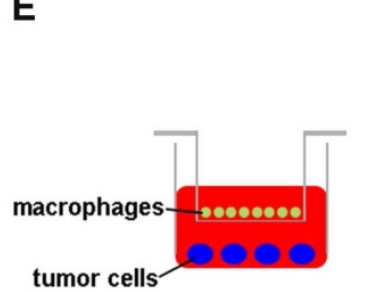

I

$\mathbf{F}$

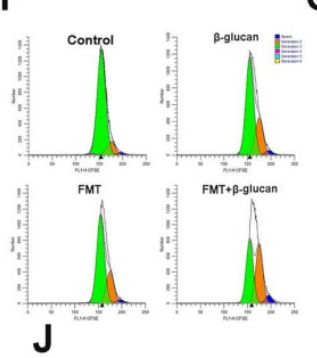

C

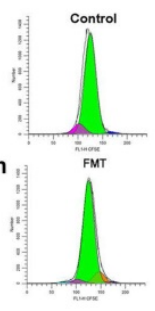

G

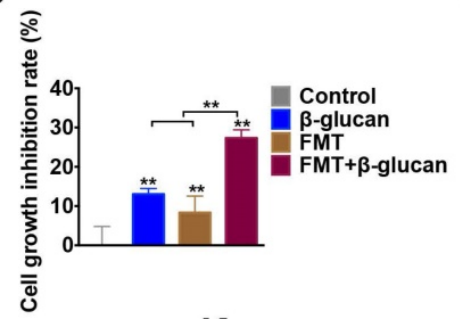

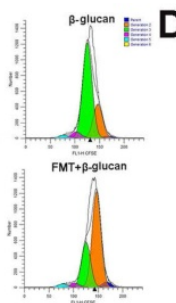

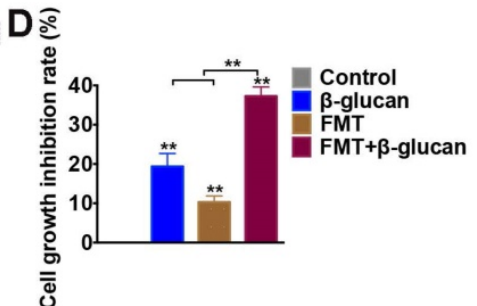

H

K
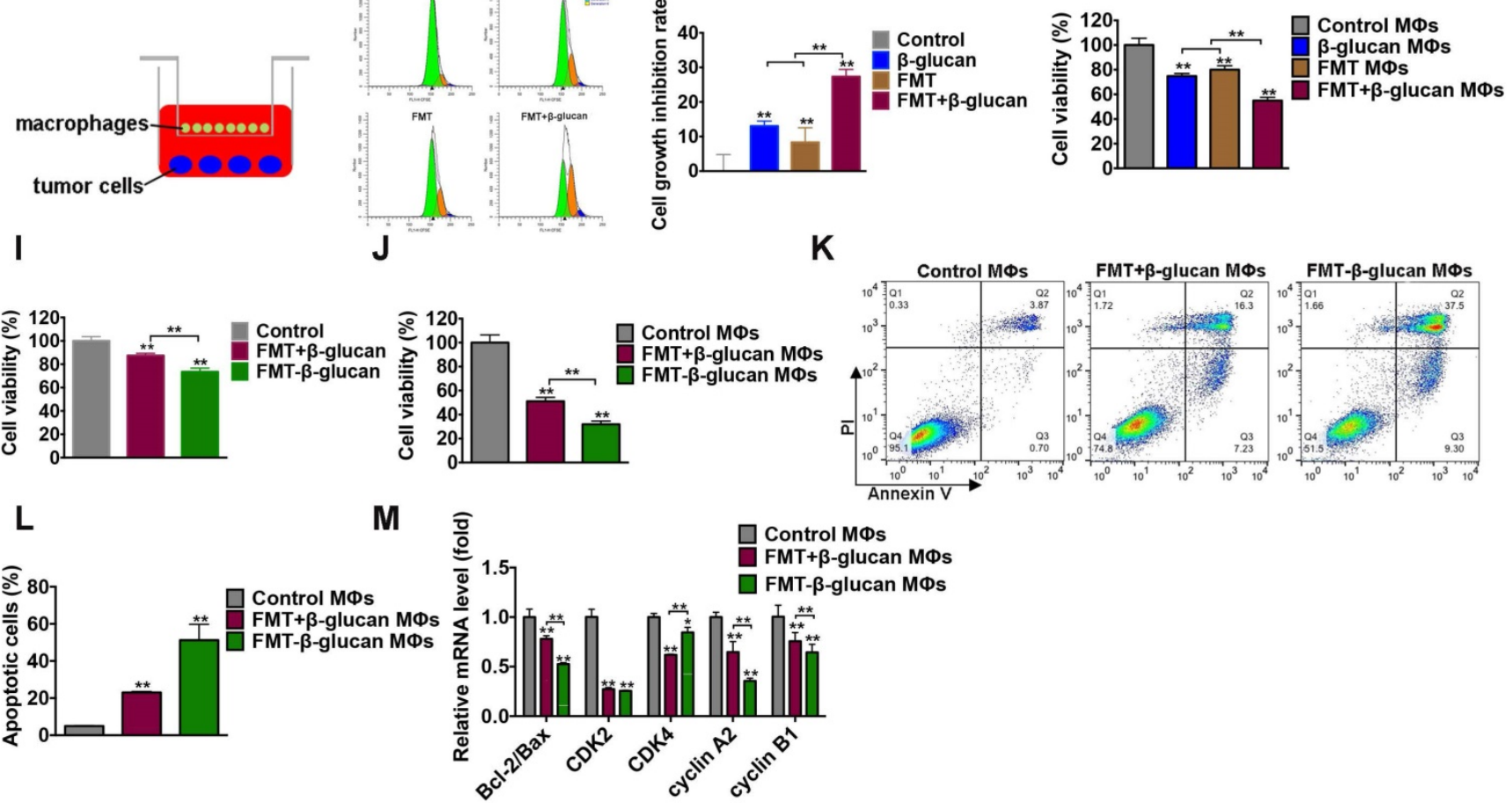

Figure 4. FMT- $\beta$-glucan inhibited tumor growth in vitro. (A) B16F10 cells were treated with FMT, $\beta$-glucan, or FMT+ $\beta$-glucan for 48 h, and the cell viability was examined by CCK-8 assay. (B) In the presence of FMT, $\beta$-glucan, or FMT+ $\beta$-glucan, B16F10 cells were directly cocultured with RAW 264.7 cells for 48 h, and the cell viability was detected by CCK-8 assay. (C) In the presence of FMT, $\beta$-glucan, or FMT+ $\beta$-glucan, B16F10 cells were directly cocultured with RAW 264.7 cells for 48 h, and the cell proliferation of B16F10 cells was detected by FCM. (D) Quantitative data of (C). (E) Schematic diagram of the indirect coculture system. (F) In the presence of FMT, $\beta$-glucan, or FMT+ $\beta$-glucan, B16F10 cells were indirectly cocultured with RAW 264.7 cells for $48 \mathrm{~h}$, and the cell proliferation of B16F10 cells was detected by FCM. (G) Quantitative data of (F). (H) After FMT, $\beta$-glucan, or FMT+ $\beta$-glucan treatment for $24 \mathrm{~h}$, the cell culture supernatants of RAW 264.7 cells were collected and used to treat B16F10 cells for $48 \mathrm{~h}$. The cell viability of B16F10 cells was detected by CCK-8 assay. (I) In the presence of FMT+ $\beta$-glucan or FMT- $\beta$-glucan, the total cell viability of B16F10 cells cocultured with RAW 264.7 cells for $48 \mathrm{~h}$ were detected by CCK-8 assay. (J) The viability of B16F10 cells was detected by CCK-8 assay after FMT+ $\beta$-glucan MФs or FMT- $\beta$-glucan MФs treatment for 48 h. (K) Apoptotic cells of B16F10 cells were examined by FCM after FMT+ $\beta$-glucan MФs or FMT- $\beta$-glucan MФs treatment. (L) Quantitative data of (K). (M) Expression of the cell cycleand apoptosis-related genes of B16F10 cells after FMT+ $\beta$-glucan MФs or FMT- $\beta$-glucan MФs treatment for 24 h was detected by $q R T-P C R$. * $P<0.05$, ** $P<0.01$.
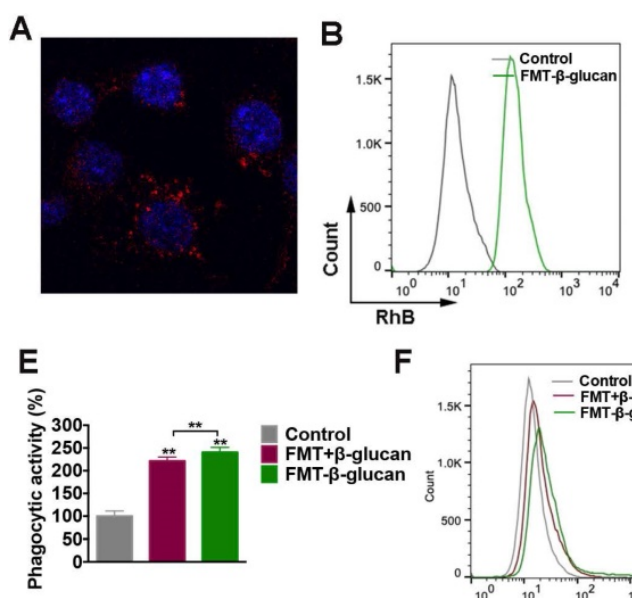

C

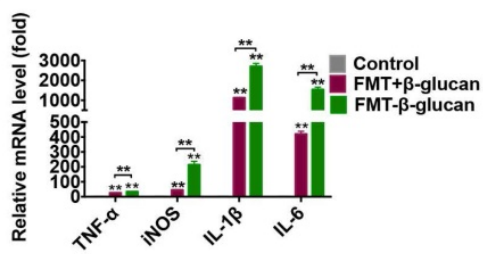

G

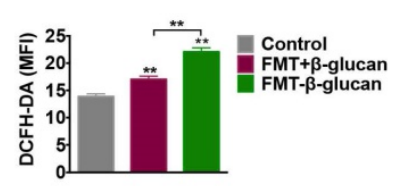

D

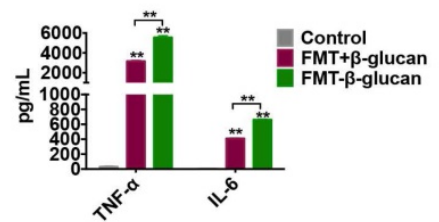

$\mathrm{H}$

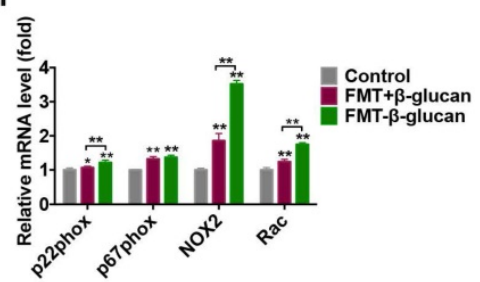

Figure 5. FMT- $\beta$-glucan promoted MI polarization, and increased phagocytosis and ROS production in macrophages. (A-B) RAW 264.7 cells were treated with FMT-B-glucan for $24 \mathrm{~h}$ or $6 \mathrm{~h}$, and the internalization of FMT- $\beta$-glucan by macrophages was detected by FV 3000 laser scanning confocal microscopy (A) and FCM (B). (C) After the treatment with FMT+ $\beta$-glucan or FMT- $\beta$-glucan for $6 \mathrm{~h}$, the M1-like gene expression in RAW 264.7 cells was detected by qRT-PCR experiment. (D) RAW 264.7 cells were 
treated with FMT+ $\beta$-glucan or FMT- $\beta$-glucan for $24 \mathrm{~h}$, and TNF- $\alpha$ and IL- 6 production in the cell culture supernatant was detected by ELISA experiment. (E) Phagocytic activities of RAW 264.7 cells were detected by the neutral red uptake assay after FMT+ $\beta$-glucan or FMT- $\beta$-glucan incubation for $24 \mathrm{~h}$. (F) RAW 264.7 cells were incubated with FMT, $\beta$-glucan or FMT+ $\beta$-glucan for $12 \mathrm{~h}$, and intracellular ROS production was detected by FCM. (G) Quantitative data of $(F)$. (H) RAW 264.7 cells were treated with FMT+ $\beta$-glucan or FMT- $\beta$-glucan for $6 \mathrm{~h}$, and the expression of p22phox, p47phox, NOX2, and Rac was detected by qRT-PCR experiment. *p $<0.05$, **p $<0.01$.

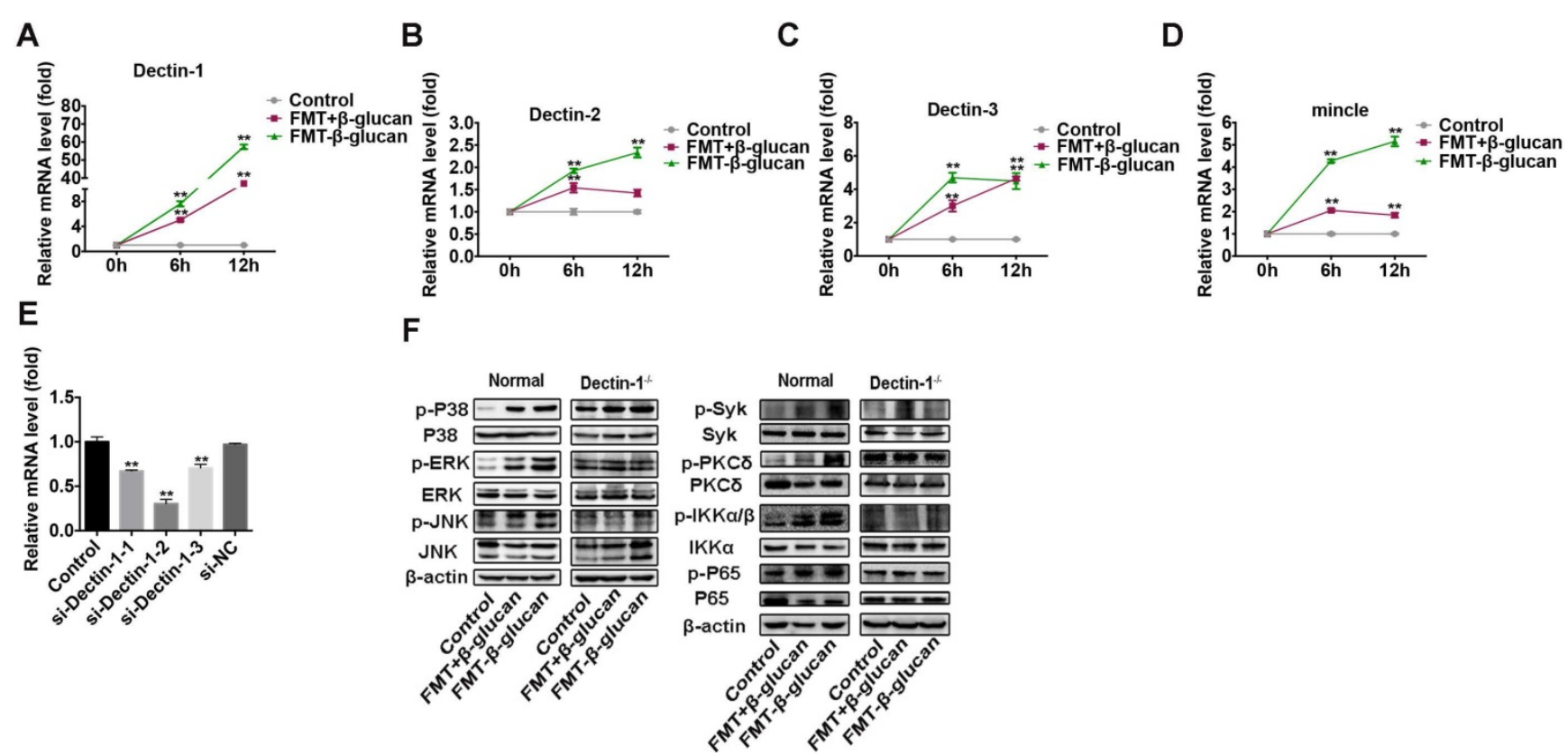

Figure 6. FMT- $\beta$-glucan activated macrophages by interacting with Dectin-1 on the surface of macrophages. (A-D) RAW 264.7 cells were incubated with FMT+ $\beta$-glucan or FMT- $\beta$-glucan for $6 \mathrm{~h}$, and the expression of Dectin-1 (A), Dectin-2 (B), Dectin-3 (C) and mincle (D) was examined by qRT-PCR assay. (E) The Dectin-1 knockdown efficacy by siRNA transfection was detected by qRT-PCR experiment. (F) RAW 264.7 cells with or without Dectin-1/- were treated with FMT+ $\beta$-glucan or FMT- $\beta$-glucan for $24 \mathrm{~h}$, and the expression of target genes was determined by WB assay. ${ }^{*} \mathrm{p}<0.05$, ${ }^{* *} \mathrm{p}<0.01$.

Previous studies have demonstrated that tumoricidal macrophages are not only characterized by M1 markers, but can also suppress cancer by engulfing tumor cells and producing reactive oxygen species (ROS) [40, 41]. The neutral red uptake assay and DCFH-DA were utilized to assess the phagocytic activity and intracellular ROS of RAW 264.7 cells. RAW 264.7 cells treated with FMT- $\beta$-glucan had an enhanced phagocytic capacity and increased intracellular ROS, which was slightly higher than that of cells treated with FMT+ $\beta$-glucan (Figure 5E-5G). Nicotinamide adenine dinucleotide phosphate (NADPH) oxidase, composed of membrane subunits (gp91phox (NOX2) and p22phox), cytoplasmic subunits (p40phox, p47phox, and p67phox) and GTPase binding protein (Rac), is the main enzyme that generates ROS [42]. Among these genes, NOX2 was upregulated to the greatest degree following costimulation (Figure 5H). Taken together, FMT- $\beta$-glucan polarized macrophages toward a tumoricidal phenotype, with upregulation of M1-like markers, phagocytic capability and intracellular ROS.

\section{FMT- $\beta$-glucan activated macrophages by interacting with Dectin-1 on the surface of macrophages}

In addition to its internalization by macrophages, FMT- $\beta$-glucan might exert its activity by binding to receptors on cells [43]. C-type lectin receptors (CLRs) are pattern recognition receptors mainly expressed on immune cell surfaces to mediate the inflammatory response. Several CLRs, such as Dectin-1, Dectin-2 and mincle, can induce signaling cascades to activate the NF-kB pathway in a Syk-dependent manner [44]. Thus, the expression of several CLRs was examined by qRT-PCR and WB assays. The results showed that Dectin-1 was upregulated to the greatest degree following FMT- $\beta$ glucan stimulation at the transcriptional level (Figure 6A-6D).

According to our investigation, FMT $+\beta$-glucan could polarize macrophages into the M1 type by activating the MAPK and Syk/NF-kB pathways (Figure S3). Whether FMT- $\beta$-glucan could activate MAPK and NF-kB pathways and Dectin-1 was involved in the phosphorylation of components in the MAPK and NF-KB pathway was unclear. To verify the role of Dectin-1, si-Dectin-1 was used to knock down the expression of Dectin-1 in RAW 264.7 cells. The knockdown efficacy was confirmed by downregulated Dectin-1 expression at the mRNA level (Figure 6E). As shown in Figure 6F, the expression of p-P38, p-ERK, and p-JNK in the MAPK pathway and p-Syk, p-PKC $\delta, p-I K K a / \beta$, and p-P65 in the Syk/NF-kB signaling were highly elevated with FMT- $\beta$-glucan stimulation. In addition, the elevated 
phosphorylated proteins in the FMT- $\beta$-glucan-treated group were greater than that those in the $\mathrm{FMT}+\beta$ glucan treatment group. Furthermore, when the Dectin-1 gene was knockdown in RAW 264.7 cells, the phosphorylation of proteins caused by FMT- $\beta$-glucan incubation was highly abrogated. Overall, Dectin-1 might play a vital role in macrophage activation induced by FMT- $\beta$-glucan.

\section{Discussion}

Tumor cells, immune and inflammatory cells, fibroblasts, and other cells constitute a complex tumor microenvironment, in which immune cells play a vital role and significantly correlated with the progression and prognosis of patients $[45,46]$. Therapeutic strategies focusing on activation or modification of immune cells have been used to inhibit melanoma growth. Interleukin (IL)-2 was approved by the FDA in 1998 for metastatic melanoma therapy and can act directly on effector $\mathrm{CD}^{+} \mathrm{T}$ and regulatory $\mathrm{CD}^{+} \mathrm{T}$ cells [47]. Due to adverse events such as tachycardia and multiple system organ failure and the poor response rate of patients with high serum vascular endothelial growth factor and fibronectin, IL-2 therapy has not been widely used [48]. In addition, chimeric antigen receptor (CAR)-T cell therapy in which $T$ cells are genetically engineered to recognize tumor antigens (melanoma-associated antigen-A3, mesenchymal epithelial transition, CD70, GD2, etc.) has also entered clinical trials in melanoma immunotherapy $[49,50]$. However, because of the self-deficiencies of CAR-T cell therapy (poor solid tumor curative effect, cytokine release syndrome, off-target effect, etc.), there was no reports of large-scale clinical treatment of melanoma by CAR-T therapy [51]. Therefore, how to effectively activate the body's own immune cells that had the potential to kill tumor cells is the key to immunotherapy in melanoma.

In the tumor milieu, infiltrated macrophages initially have an M1-polarized phenotype. The classic proinflammatory M1 type exerts antigen-presenting and tumoricidal activities, partly through expressing high levels of the major histocompatibility complex class I and II molecules, and secreting cytokines (e.g., TNF- $\alpha$, IL-1 $\beta$ and IL-6), and reactive species of oxygen and nitrogen (e.g., nitric oxide and superoxide) [52]. However, continued presence in the tumor microenvironment polarizes them to M2-like tumor-associated macrophages. The M2 type macrophages lose their ability to phagocytize or present tumor associated antigens to $\mathrm{T}$ cells [53]. Moreover, they can support cancer cell growth, silence effector immune cells, enhance angiogenesis, and promote invasion and metastasis of tumors, resulting partly from producing various mediators (tumor growth factor- $\beta$, prostaglandin E2, vascular endothelial growth factor, etc.) and enzymes (metalloproteinase 2, cathepsins, etc.) [54, 55]. In melanoma, tumor-associated macrophages account for the majority of all immune cells. Therefore, polarizing tumor-associated macrophages into the antitumor M1 type could be a beneficial method to effectively treat melanoma.

Nanoparticles, generally characterized by submicronic $(<1 \mu \mathrm{m})$ systems of metallic or organic materials, offer broad applications in various fields including tumor detection, imaging, diagnosis and treatment [56]. Several types of nanoparticles, such as carbonaceous, Prussian blue, or paramagnetic nanoparticles are available for in vitro diagnosis based on their peroxidase-like activity or function as enzyme carriers $[57,58]$ and in vivo diagnosis as carriers of contrast agents [59, 60]. In addition to diagnosis, their current applications in cancer treatment have gained widespread interest. Numerous investigations have shown that nanoparticles can control the distribution of anticancer drugs via a drug vehicle able to target tumor tissues or cells $[61,62]$. However, due to inappropriate pharmacokinetic properties and concerns about biodegradation, elimination and toxicity, most nanoparticles can be applied only to nanodiagnostics and not to nanotherapeutics [63]. Because the release of catalytic free iron from FMT was negligible, FMT was very suitable to select as a carrier of tumor therapy agents in this study with no toxicity.

Chemotherapeutic drugs, such as doxorubicin and paclitaxel can be bound to the surface of nanoparticles and delivered to tumor sites with increased accumulation in pathological organs and protection from nonspecific toxicity [64, 65]. Moreover, surface-modified nanoparticles could be rapidly opsonized and massively endocytosed/ phagocytosed by cells from the mononuclear phagocyte system (bone marrow, spleen, liver, and lungs) after systemic administration, resulting in modulation of the tumor microenvironment [66]. As an important participant in the tumor microenvironment, tumor-associated macrophages (TAMs) with an M2-like profile could confer to an immunosuppressive state and further promote tumor progression [67]. Changing TAMs from the M2 to M1 type may provide a more effective tumor therapeutic strategy. In addition to the role of drug carriers, FMT was shown to induce macrophages into the M1 type and exerted antitumor activity in our study, which was consistent with other studies [68]. After FMT stimulation, macrophages tend to develop into 
M1-type macrophages, accompanied by the increased expression of M1-related genes and cytokines (TNF- $\alpha$ and IL-6). In the presence of FMT, macrophages significantly reduced the tumor cell viability of tumor cells when directly and indirectly cocultured with tumor cells. In general, FMT can not only deliver immune activators, but can also have a synergistic effect when used in combination with other activators. $\beta$-glucan receptors including complement receptor 3 (TLR 3) and Dectin-1, are widely expressed on myeloid cells [69]. By binding to these receptors, $\beta$-glucan exerts its immunomodulating effects to defend against infection and prevent tumor growth by activating various immune cells (macrophages, $\mathrm{T}$ cells, natural killer cells, etc.) [70, 71]. Consistent with these studies, we found that solo treatment with $\beta$-glucan could indirectly inhibit B16F10 cell growth by skewing macrophages toward an M1 phenotype through activating MAPK and NF-kB signaling.

In this study, we aimed to prepare a nanocomposite of FMT- $\beta$-glucan and explore whether FMT- $\beta$-glucan had the same or better effect on immune regulation and tumor inhibition than the combination treatment of FMT plus $\beta$-glucan. Compared with $\mathrm{FMT}+\beta$-glucan, intraperitoneal administration of FMT- $\beta$-glucan to male B16F10 bearing mice more strongly suppressed tumor growth and induced increased infiltration of M1-like macrophages in tumor tissues. In vitro mechanistic studies indicated that FMT- $\beta$-glucan might polarize macrophages into M1-type macrophages by binding to Dectin-1 on the surface of RAW 264.7 cells to exert a stronger toxicity to tumor cells. Collectively, this study provides a novel way to activate the immune system to maximize the efficacy of cancer therapy.

\section{Conclusion}

In this study, a macrophage-targeted nanocomposite for melanoma therapy was synthesized. Taking advantage of the carrier function of FMT and the immune regulation of $\beta$-glucan, the nanocomposite of FMT- $\beta$-glucan displayed better anti-melanoma efficacy than the combined treatment of FMT plus $\beta$-glucan by inducing the M1 macrophage-activated tumor microenvironment. Further studies showed that FMT was engulfed by macrophages and activated the MAPK and Syk/NF-kB pathways to polarize macrophages into M1 type through the Dectin-1 receptor. M1-like macrophages induced apoptosis and cell cycle arrest of tumor cells by releasing multiple factors including TNF- $\alpha$, IL- 6 and ROS. Our results suggested that FMT- $\beta$-glucan might hold great promise for the clinical management of melanoma.

\section{Abbreviations}

FMT: Ferumoxytol; IL-2: Interleukin-2; CAR-T: chimeric antigen receptor-T; TNF- $\alpha$ : tumor necrosis factor- $\alpha$; TLR 3: toll-like receptor 3; FMT surface-coated with $\beta$-glucan: FMT- $\beta$-glucan; MAPK: mitogen-activated protein kinase; Syk: spleen associated tyrosine kinase; PKC $\delta$ : protein kinase $C$ delta; NF-кB: nuclear factor kappa-B; DMEM: Dulbecco's modified Eagle's medium; M-CSF: macrophage colony-stimulating factor; CCK-8: Cell Counting Kit-8; qRT-PCR: quantitative real timepolymerase chain reaction; ELISA: enzyme linked immunosorbent assay; PBS: phosphate buffered solution; ROS: reactive oxygen species; DCFH-DA: 2,7-Dichlorodi-hydrofluorescein diacetate; FCM: flow cytometry; WB: Western blotting; BSA: bovine serum albumin; TBST: Tris-buffered saline with $0.1 \%$ Tween 20; iNOS: inducible nitric oxide synthase; RhB: Rhodamine B; DAPI: 4,6-diamino-2-phenyl-indole; siRNA: small interfering RNA; IHC: Immunohistochemistry; NADPH: Nicotinamide adenine dinucleotide phosphate; DLS: Dynamic light scattering; TAMs: tumor-associated macrophages; M-MDSCs: myeloid-derived suppressor cells.

\section{Supplementary Material}

Supplementary figures.

http://www.medsci.org/v18p3125s1.pdf

\section{Acknowledgements}

This work was supported by grant from Key Research and Development Program of Jiangsu Province (grant number: BE2019617).

\section{Author Contributions}

Xinghan Liu designed and performed the experiments, Yujun $\mathrm{Xu}, \mathrm{Yi} \mathrm{Li}$, and Yuchen Pan helped the experiments and analysed the data, Shuli Zhao and Yayi Hou coordinated the project and provided suggestions to the experiments.

\section{Competing Interests}

The authors have declared that no competing interest exists.

\section{References}

1. Siegel RL, Miller KD, Jemal A. Cancer statistics, 2020. CA: a cancer journal for clinicians. 2020; 70: 7-30.

2. Che G, Huang B, Xie Z, et al. Trends in incidence and survival in patients with melanoma, 1974-2013. American journal of cancer research. 2019; 9: 1396-1414.

3. Sandru A, Voinea S, Panaitescu E, et al. Survival rates of patients with metastatic malignant melanoma. Journal of medicine and life. 2014; 7: 572-576.

4. Antohe M, Nedelcu RI, Nichita L, et al. Tumor infiltrating lymphocytes: the regulator of melanoma evolution. Oncology letters. 2019; 17: 4155-4161.

5. Cuevas LM, Daud AI. Immunotherapy for melanoma. Seminars in cutaneous edicine and surgery. 2018; 37: 127-131.

6. Robert C, Ribas A, Schachter J, et al. Pembrolizumab versus ipilimumab in advanced melanoma (KEYNOTE-006): post-hoc 5-year results from an 
open-label, multicentre, randomised, controlled, phase 3 study. The Lancet Oncology. 2019; 20: 1239-1251.

7. Khushalani NI, Diab A, Ascierto PA, et al. Bempegaldesleukin plus nivolumab in untreated, unresectable or metastatic melanoma: Phase III PIVOT IO 001 study design. Future oncology (London, England). 2020; 16: 2165-2175.

8. O'Donnell JS, Teng MWL, Smyth MJ. Cancer immunoediting and resistance to T cell-based immunotherapy. Nature reviews Clinical oncology. 2019; 16: 151-167.

9. Winder M, Virós A. Mechanisms of drug resistance in melanoma. Handbook of experimental pharmacology. 2018; 249: 91-108.

10. Lu M, Cohen MH, Rieves D, et al. FDA report: Ferumoxytol for intravenous iron therapy in adult patients with chronic kidney disease. American journal of hematology. 2010; 85: 315-319.

11. Auerbach M, Chertow GM, Rosner M. Ferumoxytol for the treatment of iron deficiency anemia. Expert review of hematology. 2018; 11: 829-834.

12. Spinowitz BS, Kausz AT, Baptista J, et al. Ferumoxytol for treating iron deficiency anemia in CKD. Journal of the American Society of Nephrology: JASN. 2008; 19: 1599-1605.

13. Shimizu K, Kushamae M, Aoki T. Macrophage imaging of intracranial aneurysms. Neurologia medico-chirurgica. 2019; 59: 257-263.

14. Morishige K, Kacher DF, Libby P, et al. High-resolution magnetic resonance imaging enhanced with superparamagnetic nanoparticles measures macrophage burden in atherosclerosis. Circulation. 2010; 122: 1707-1715.

15. Zanganeh S, Hutter G, Spitler R, et al. Iron oxide nanoparticles inhibit tumour growth by inducing pro-inflammatory macrophage polarization in tumour tissues. Nature nanotechnology. 2016; 11: 986-994.

16. Zhao J, Zhang Z, Xue $\mathrm{Y}$, et al. Anti-tumor macrophages activated by ferumoxytol combined or surface-functionalized with the TLR3 agonist poly (I:C) promote melanoma regression. Theranostics. 2018; 8: 6307-6321.

17. Wang G, Zhao J, Zhang M, et al. Ferumoxytol and CpG oligodeoxynucleotide 2395 synergistically enhance antitumor activity of macrophages against NSCLC with EGFR(L858R/T790M) mutation. International journal of nanomedicine. 2019; 14: 4503-4515.

18. Nakashima A, Yamada $K$, Iwata $O$, et al. $\beta$-glucan in foods and its physiological functions. J Nutr Sci Vitaminol (Tokyo). 2018; 64: 8-17.

19. Brown GD, Gordon S. Fungal beta-glucans and mammalian immunity. Immunity. 2003; 19: 311-315.

20. Synytsya A, Novák M. Structural diversity of fungal glucans. Carbohydrate polymers. 2013; 92: 792-809.

21. Geller A, Shrestha R, Yan J. Yeast-derived $\beta$-glucan in cancer: novel uses of a traditional therapeutic. International journal of molecular sciences. 2019; 20: 3618 .

22. Akramiene D, Kondrotas A, Didziapetriene J, et al. Effects of beta-glucans on the immune system. Medicina (Kaunas). 2007; 43: 597-606.

23. Baert K, Sonck E, Goddeeris BM, et al. Cell type-specific differences in $\beta$-glucan recognition and signalling in porcine innate immune cells. Dev Comp Immunol. 2015; 48: 192-203.

24. Li B, Allendorf DJ, Hansen R, et al. Yeast beta-glucan amplifies phagocyte killing of iC3b-opsonized tumor cells via complement receptor 3-Sykphosphatidylinositol 3-kinase pathway. Journal of immunology (Baltimore, Md:1950). 2006; 177: 1661-1669.

25. Vetvicka V, Vetvickova J. Glucan supplementation has strong anti-melanoma effects: role of NK cells. Anticancer research. 2015; 35: 5287-5292.

26. Albeituni SH, Ding $\mathrm{C}$, Liu $\mathrm{M}$, et al. Yeast-derived particulate $\beta$-glucan treatment subverts the suppression of myeloid-derived suppressor cells (MDSC) by inducing polymorphonuclear MDSC apoptosis and monocytic MDSC differentiation to APC in cancer. Journal of immunology (Baltimore, Md:1950). 2016; 196: 2167-2180.

27. Liu M, Luo F, Ding C, et al. Dectin- 1 activation by a natural product $\beta$-glucan converts immunosuppressive macrophages into an M1-like phenotype. Journal of immunology (Baltimore, Md: 1950). 2015; 195: 5055-5065.

28. Cheng H, Sun L, Shen D, et al. Beta-1,6 glucan converts tumor-associated macrophages into an M1-like phenotype. Carbohydrate polymers. 2020; 247: 116715.

29. Kalafati L, Kourtzelis I, Schulte-Schrepping J, et al. Innate immune training of granulopoiesis promotes anti-tumor activity. Cell. 2020; 183: 771-785.e712.

30. Wang N, Liu H, Liu G, et al. Yeast $\beta$-D-glucan exerts antitumour activity in liver cancer through impairing autophagy and lysosomal function, promoting reactive oxygen species production and apoptosis. Redox biology. 2020; 32: 101495.

31. Moorlag S, Khan N, Novakovic B, et al. $\beta$-glucan induces protective trained immunity against mycobacterium tuberculosis infection: a key role for IL-1. Cell reports. 2020; 31: 107634 .

32. Torosantucci A, Bromuro C, Chiani P, et al. A novel glyco-conjugate vaccine against fungal pathogens. The Journal of experimental medicine. 2005; 202: 597-606.

33. Bacon JS, Farmer VC, Jones D, et al. The glucan components of the cell wall of baker's yeast (Saccharomyces cerevisiae) considered in relation to its ultrastructure. The Biochemical journal. 1969; 114: 557-567.

34. Chen B, Sun J, Fan F, et al. Ferumoxytol of ultrahigh magnetization produced by hydrocooling and magnetically internal heating co-precipitation. Nanoscale. 2018; 10: 7369-7376.

35. Liu X, Xu Y, Li Y, et al. Ganoderma lucidum fruiting body extracts inhibit colorectal cancer by inducing apoptosis, autophagy, and G0/G1 phase cell cycle arrest in vitro and in vivo. American journal of translational research. 2020; 12: 2675-2684

36. Chen S, Yin $\mathrm{L}, \mathrm{Xu} \mathrm{Z}$, et al. Inhibiting receptor for advanced glycation end product (AGE) and oxidative stress involved in the protective effect mediated by glucagon-like peptide-1 receptor on AGE induced neuronal apoptosis. Neuroscience letters. 2016; 612: 193-198.

37. Schafer KA. The cell cycle: a review. Veterinary pathology. 1998; 35: 461-478.

38. Pistritto G, Trisciuoglio D, Ceci C, et al. Apoptosis as anticancer mechanism: function and dysfunction of its modulators and targeted therapeutic strategies. Aging. 2016; 8: 603-619.

39. Juhas U, Ryba-Stanisławowska M, Szargiej P, et al. Different pathways of macrophage activation and polarization. Postepy Hig Med Dosw (Online). 2015; 69: 496-502.

40. Forman HJ, Torres M. Reactive oxygen species and cell signaling: respiratory burst in macrophage signaling. American journal of respiratory and critical care medicine. 2002; 166: S4-8

41. Devi KS, Behera B, Mishra D, et al. Immune augmentation and Dalton's lymphoma tumor inhibition by glucans/glycans isolated from the mycelia and fruit body of Pleurotus ostreatus. International immunopharmacology. 2015; 25: 207-217.

42. Xu Q, Choksi S, Qu J, et al. NADPH oxidases are essential for macrophage differentiation. The Journal of biological chemistry. 2016; 291: 20030-20041.

43. Yang Y, Zhao X, Li J, et al. A $\beta$-glucan from durvillaea antarctica has immunomodulatory effects on RAW264.7 macrophages via toll-like receptor 4 . Carbohydrate polymers. 2018; 191: 255-265.

44. Kingeter LM, Lin X. C-type lectin receptor-induced NF-kB activation in innate immune and inflammatory responses. Cellular \& molecular immunology. 2012; 9: 105-112.

45. Marzagalli M, Ebelt ND, Manuel ER. Unraveling the crosstalk between melanoma and immune cells in the tumor microenvironment. Seminars in cancer biology. 2019; 59: 236-250.

46. Lee N, Zakka LR, Mihm MC, Jr., et al. Tumour-infiltrating lymphocytes in melanoma prognosis and cancer immunotherapy. Pathology. 2016; 48: 177-187.

47. Krieg C, Létourneau S, Pantaleo G, et al. Improved IL-2 immunotherapy by selective stimulation of IL-2 receptors on lymphocytes and endothelial cells. Proc Natl Acad Sci U S A. 2010; 107: 11906-11911.

48. Sabatino M, Kim-Schulze S, Panelli MC, et al. Serum vascular endothelial growth factor and fibronectin predict clinical response to high-dose interleukin-2 therapy. Journal of clinical oncology: official journal of the American Society of Clinical Oncology. 2009; 27: 2645-2652.

49. Lu YC, Parker LL, Lu T, et al. Treatment of patients with metastatic cancer using a major histocompatibility complex class II-restricted T-cell receptor targeting the cancer germline antigen MAGE-A3. Journal of clinical oncology: official journal of the American Society of Clinical Oncology. 2017; 35: 3322-3329.

50. Simon B, Uslu U. CAR-T cell therapy in melanoma: a future success story? Experimental dermatology. 2018; 27: 1315-1321.

51. Yáñez L, Alarcón A, Sánchez-Escamilla M, et al. How I treat adverse effects of CAR-T cell therapy. ESMO open. 2020; 4

52. Martinez FO, Sica A, Mantovani A, et al. Macrophage activation and polarization. Front Biosci. 2008; 13: 453-461.

53. Chanmee T, Ontong P, Konno K, et al. Tumor-associated macrophages as major players in the tumor microenvironment. Cancers. 2014; 6: 1670-1690.

54. Pieniazek M, Matkowski R, Donizy P. Macrophages in skin melanoma-the key element in melanomagenesis. Oncology letters. 2018; 15: 5399-5404.

55. Nasrollahzadeh E, Razi S, Keshavarz-Fathi M, et al. Pro-tumorigenic functions of macrophages at the primary, invasive and metastatic tumor site. Cancer immunology, immunotherapy: CII. 2020; 69: 1673-1697.

56. Brigger I, Dubernet C, Couvreur P. Nanoparticles in cancer therapy and diagnosis. Advanced drug delivery reviews. 2002; 54: 631-651.

57. Wang Y, Xia Y. Optical, electrochemical and catalytic methods for in-vitro diagnosis using carbonaceous nanoparticles: a review. Mikrochimica acta. 2019; 186: 50.

58. Martinkova P, Pohanka M. Phone camera detection of glucose blood level based on magnetic particles entrapped inside bubble wrap. Neuro endocrinology letters. 2016; 37: 132-138.

59. Jayapaul J, Schröder L. Nanoparticle-based contrast agents for (129)Xe HyperCEST NMR and MRI applications. Contrast media \& molecular imaging. 2019; 2019: 9498173

60. Wang X, Wang J, Pan J, et al. Rhenium sulfide nanoparticles as a biosafe spectral CT contrast agent for gastrointestinal tract imaging and tumor theranostics in vivo. ACS applied materials \& interfaces. 2019; 11: 33650-33658.

61. De Jong WH, Borm PJ. Drug delivery and nanoparticles:applications and hazards. International journal of nanomedicine. 2008; 3: 133-149.

62. Nguyen-Ngoc T, Raymond E. Reinvention of chemotherapy: drug conjugates and nanoparticles. Current opinion in oncology. 2015; 27: 232-242.

63. Baetke SC, Lammers T, Kiessling F. Applications of nanoparticles for diagnosis and therapy of cancer. The British journal of radiology. 2015; 88: 20150207

64. Win KY, Feng SS. In vitro and in vivo studies on vitamin E TPGS-emulsified poly(D,L-lactic-co-glycolic acid) nanoparticles for paclitaxel formulation. Biomaterials. 2006; 27: 2285-2291. 
65. Park J, Fong PM, Lu J, et al. PEGylated PLGA nanoparticles for the improved delivery of doxorubicin. Nanomedicine: nanotechnology, biology, and medicine. 2009; 5: 410-418.

66. Yang S, Gao H. Nanoparticles for modulating tumor microenvironment to improve drug delivery and tumor therapy. Pharmacological research. 2017; 126: 97-108.

67. Saccani A, Schioppa T, Porta C, et al. P50 nuclear factor-kappaB overexpression in tumor-associated macrophages inhibits M1 inflammatory responses and antitumor resistance. Cancer research. 2006; 66: 11432-11440.

68. Wang Y, Lin YX, Qiao SL, et al. Polymeric nanoparticles promote macrophage reversal from M2 to M1 phenotypes in the tumor microenvironment. Biomaterials. 2017; 112: 153-163.

69. Goodridge HS, Wolf AJ, Underhill DM. Beta-glucan recognition by the innate immune system. Immunological reviews. 2009; 230: 38-50.

70. Zhang M, Chun L, Sandoval V, et al. Systemic administration of $\beta$-glucan of $200 \mathrm{kDa}$ modulates melanoma microenvironment and suppresses metastatic cancer. Oncoimmunology. 2018; 7: e1387347.

71. Chan GC, Chan WK, Sze DM. The effects of beta-glucan on human immune and cancer cells. J Hematol Oncol. 2009; 2: 25. 